\title{
L'habitat du Néolithique ancien de Fontenay-le- Marmion « Le Grand Champ / Le chemin Haussé »
}

The Early Neolithic Habitation at Fontenay-le-Marmion "Le Grand Champ/Le Chemin Haussé (Calvados)

Pierre Giraud, Laurent Juhel, Caroline Hamon, Cécile Germain, Xavier Savary, Denis Jan et Avec les contributions de Cécile Riquier et de JeanPaul Caspar ( $(\mathbf{)}$

\section{(2) OpenEdition}

\section{Journals}

Édition électronique

URL : https://journals.openedition.org/rao/1715

DOI : 10.4000/rao.1715

ISBN : 978-2-7535-2790-4

ISSN : 1775-3732

Éditeur

Presses universitaires de Rennes

Édition imprimée

Date de publication : 30 décembre 2012

Pagination : 51-89

ISBN : 978-2-7535-2641-9

ISSN : 0767-709X

Référence électronique

Pierre Giraud, Laurent Juhel, Caroline Hamon, Cécile Germain, Xavier Savary, Denis Jan et Avec les contributions de Cécile Riquier et de Jean-Paul Caspar (†), « L'habitat du Néolithique ancien de Fontenay-le-Marmion "Le Grand Champ / Le chemin Haussé » », Revue archéologique de l'Ouest [En ligne], 29 | 2012, mis en ligne le 30 décembre 2014, consulté le 22 août 2022. URL : http:// journals.openedition.org/rao/1715; DOI : https://doi.org/10.4000/rao.1715 


\section{L'habitat du Néolithique ancien de Fontenay-le-Marmion « Le Grand Champ / Le chemin Haussé »}

The Early Neolithic Habitation at Fontenay-le-Marmion "Le Grand Champ/Le Chemin Haussé (Calvados)

Pierre Giraud, Laurent Juhel, Caroline Hamon, Cécile Germain, Xavier Savary, Denis Jan et Avec les contributions de Cécile Riquier et de JeanPaul Caspar (t)

\section{NOTE DE L'ÉDITEUR}

Manuscrit proposé le 25 aout 2011, accepté le 30 novembre 2011.

\section{Introduction}

\section{Présentation}

$1 \quad$ Il s'agit d'une opération de fouilles préventives réalisée, du 5 janvier 2004 au 31 mars 2004, dans le cadre de la construction de la déviation de la RD 562. Le site est localisé en limite orientale du Bassin parisien, sur le plateau calcaire faiblement ondulé de la plaine de Caen, à une dizaine de kilomètres au sud de l'agglomération caennaise (fig. 1). Ce plateau calcaire est surmonté par une couverture lœssique discontinue. À hauteur du site de Fontenay-le-Marmion, cette couverture lœssique atteint une épaisseur de quatre-vingts centimètres à un mètre. La vallée de la Laize qui marque la limite occidentale du Massif armoricain avec l'apparition des formations de grès schisteux, est localisée à deux kilomètres à l'est du site (fig. 2). 
Figure 1 : Localisation du site sur le territoire du département du Calvados. Figure 1: Site localisation in the Calvados department (Lower-Normandy).

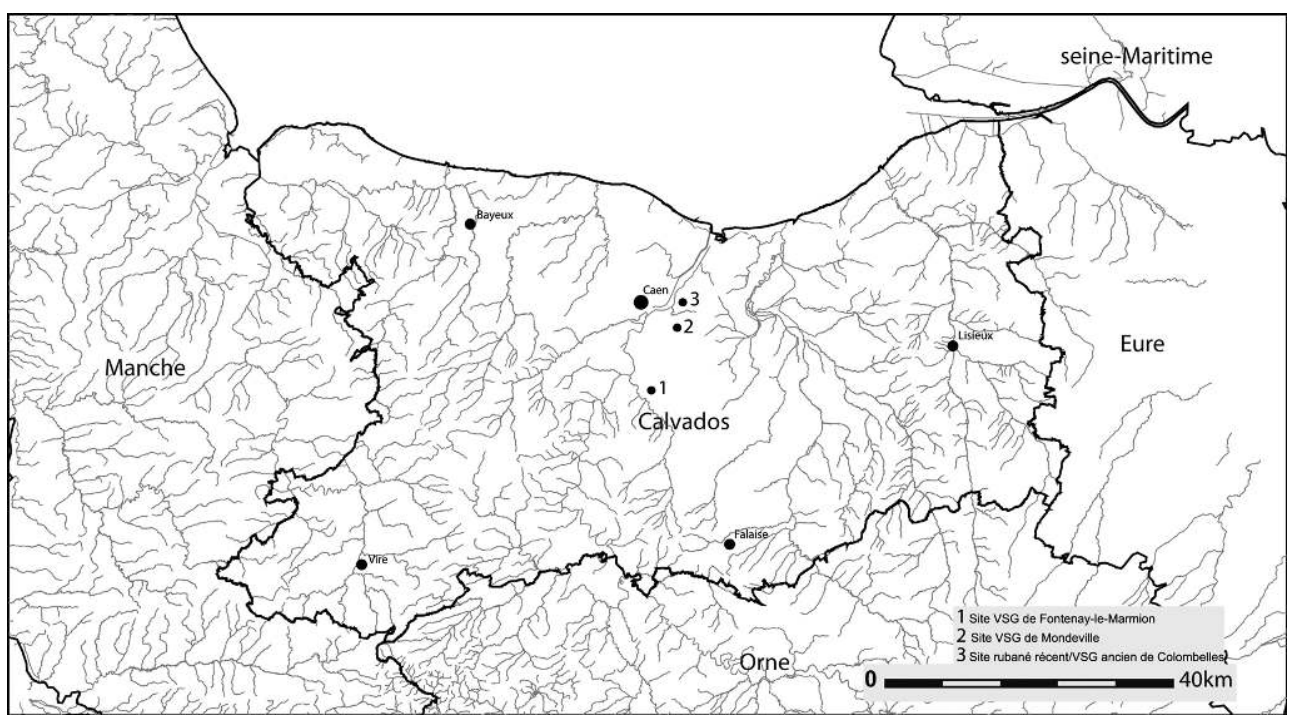

Figure 2 : Localisation du site sur orthophotoplan. CG 14. Figure 2: Site localisation on an orthophoto map.

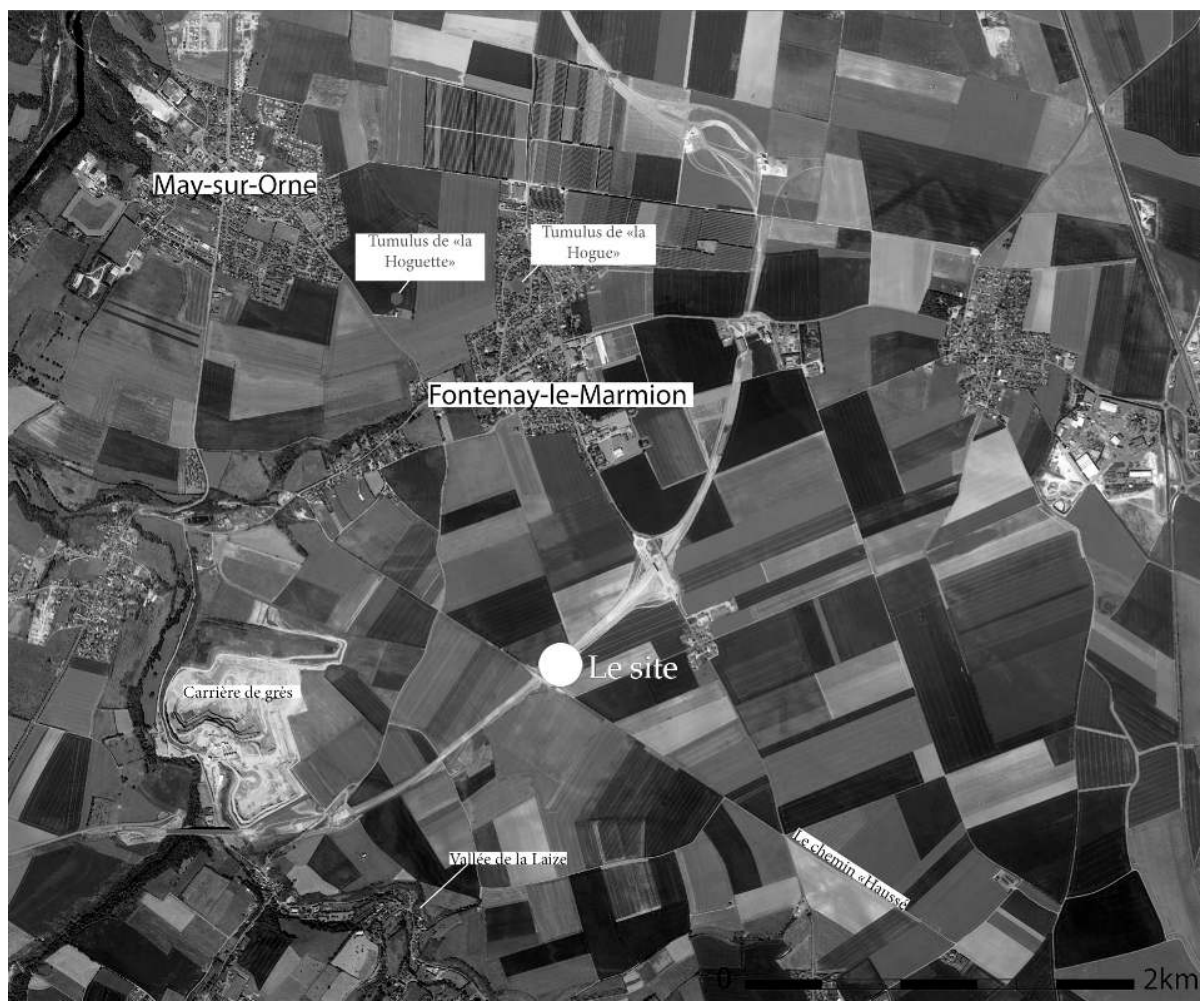

Onze sites du Néolithique ont été répertoriés sur la commune de Fontenay-le-Marmion. Parmi eux, le tumulus en pierres sèches de La Hoguette qui comprend sept à huit chambres à couloir dans lesquelles a été trouvée une soixantaine d'individus. Ce monument est daté par analyse radiocarbone entre 5000 et 3050 BC. Les autres sites inventoriés dans la carte archéologique de la commune sont des stations de surface, certaines sont localisées à proximité immédiate de cet habitat. 


\section{Méthodes de travail}

3 La principale difficulté de cette opération est la mauvaise lisibilité des structures dont les limites ne se distinguent pratiquement pas du sédiment en place.

4 Nous avons réalisé un décapage par passes très fines jusqu'au niveau d'apparition du mobilier, ou celui de traces d'éventuelles structures. Un deuxième décapage, effectué également par passes très fines en fin d'opération, a permis d'atteindre le substrat calcaire sur toute la surface étudiée. Plusieurs tranchées ont été creusées à la suite du deuxième décapage dans l'objectif de compléter certaines données archéologiques et géomorphologiques.

5 Nous avons installé un carroyage métrique dans la zone comprenant les structures néolithiques (de A à AP et de 016 à 23). Des bornes de référence ont été implantées sur les autres secteurs. Les artefacts ont été localisés à l'intérieur de leur carré de fouille à l'aide de fiche au $1 / 20^{\mathrm{e}}$, par passes ou US artificiels de $10 \mathrm{~cm}$ (US 1 correspondant aux dix premiers centimètres, l'US 2 aux dix centimètres suivants, etc.). L'objectif de cette méthode est d'obtenir une répartition du mobilier à une précision de moins d'un quart de mètre carré pour l'horizontale et du décimètre pour la verticale.

6 Pour la réalisation de la fouille des fosses de l'habitat du Néolithique, nous avons utilisé deux méthodes en raison de la difficulté à identifier les limites des structures. La première, appliquée aux portions de fosses les plus lisibles, a consisté à suivre les bords identifiés de la structure, après le relevé de coupes au niveau des tranchées du diagnostic. La deuxième technique correspond à la fouille de carrés de deux mètres sur deux, en quinconce, jusqu'au substrat, de façon à obtenir un maximum de coupes dans les deux orientations. Ces deux méthodes ont leurs avantages et leurs inconvénients. Avec la première, il est parfois complexe de lire les limites des fosses; les bords mis au jour sont parfois approximatifs, mais le choix de l'orientation des coupes est plus souple que pour la seconde méthode. Cette dernière permet de multiplier les coupes et donne une meilleure lecture jusqu'au substrat, mais elle a l'inconvénient de fournir une documentation plus difficile à gérer et ne restitue pas de vues générales de la structure fouillée ; la réalisation de plans devient plus laborieuse.

\section{Contexte pédo-sédimentaire}

7 À hauteur du site de Fontenay-le-Marmion, aux dépens de la totalité de la couverture lœssique s'est développée un sol brun lessivé ou «luvisol ». Ce type de sol s'est formé suite à la décarbonatation du lœess depuis le début de l'Holocène, sous un couvert végétal protecteur et continu (Fedoroff et Courty, 1994). De ce luvisol n'est conservé aujourd'hui que l'horizon illuvial $(\mathrm{Bt})$ épais de trente à soixante centimètres. Cet horizon limoneux à limono-argileux brun orangé apparaît directement sous l'horizon de labour limoneux brun de trente à quarante centimètres d'épaisseur. Dans les vingt à trente centimètres supérieurs de l'horizon $\mathrm{Bt}$, de couleur plus brune, la présence de mobilier attribuable à la période historique suggère son remaniement, soit par colluvionnement, soit par une pédogenèse historique.

Les structures néolithiques mises au jour sur ce site sont excavées dans l'horizon illuvial Bt sous le niveau remanié de Bt repérés, grâce à la présence du mobilier historique. Elles ont été localisées à la surface de décapage par la couleur plus sombre de leur remplissage ou grâce à la présence de matériel archéologique. 


\section{Descriptions des structures}

9 Les deux décapages ont révélé qu'une seule unité d'habitation était conservée, comprenant une fosse attenante nord à deux lobes et deux fosses sud dont l'une mesure dix-sept mètres de longueur (fig. 3). Certaines excavations découvertes lors du deuxième décapage pourraient correspondre à des trous de poteaux de la maison. D'autres faits, relevés au cours de la fouille, n'ont pu être attribués à une période précise.

Figure 3 : Plan des structures. Figure 3: Plan of the features.

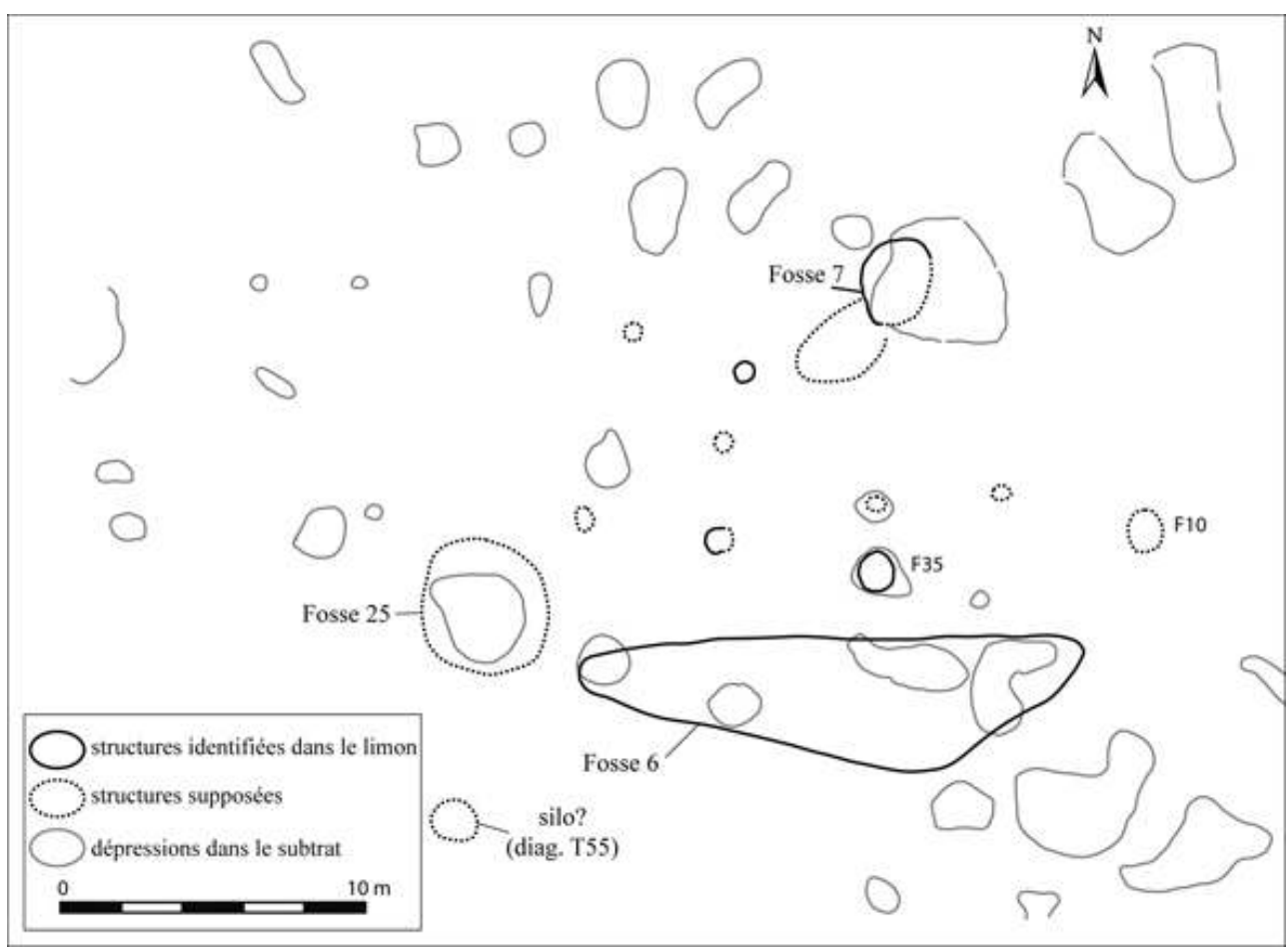

\section{Les fosses attenantes}

\section{La fosse nord (F7)}

Les deux excavations identifiées, lors du diagnostic, correspondent à deux lobes d'une même fosse (fig. 4). Le niveau de décapage de cette structure est inférieur de quinze centimètres à celui des fosses sud, car il correspond à celui d'une fenêtre d'évaluation réalisée lors du diagnostic. Le remplissage argilo-limoneux gris-jaune de la fosse nord est plus marqué dans sa partie nord où les charbons de bois sont plus nombreux. Seuls les bords du lobe nord ont pu être repérés lors de la fouille. Ils dessinent une excavation de forme plus ou moins circulaire, de deux à trois mètres de diamètre, à fond assez plat, conservé sur environ quarante centimètres de profondeur. La seconde partie (sud) pourrait être plus ovalaire, et plus profonde: quarante à cinquante centimètres d'après les indications données par la répartition du mobilier. Nous 
pouvons estimer sa taille à deux mètres cinquante de longueur pour une largeur d'un peu plus de un mètre cinquante.

Figure 4 : Vue de la fosse nord (fosse 7 ).

Figure 4: View of the northern pit (pit 7).

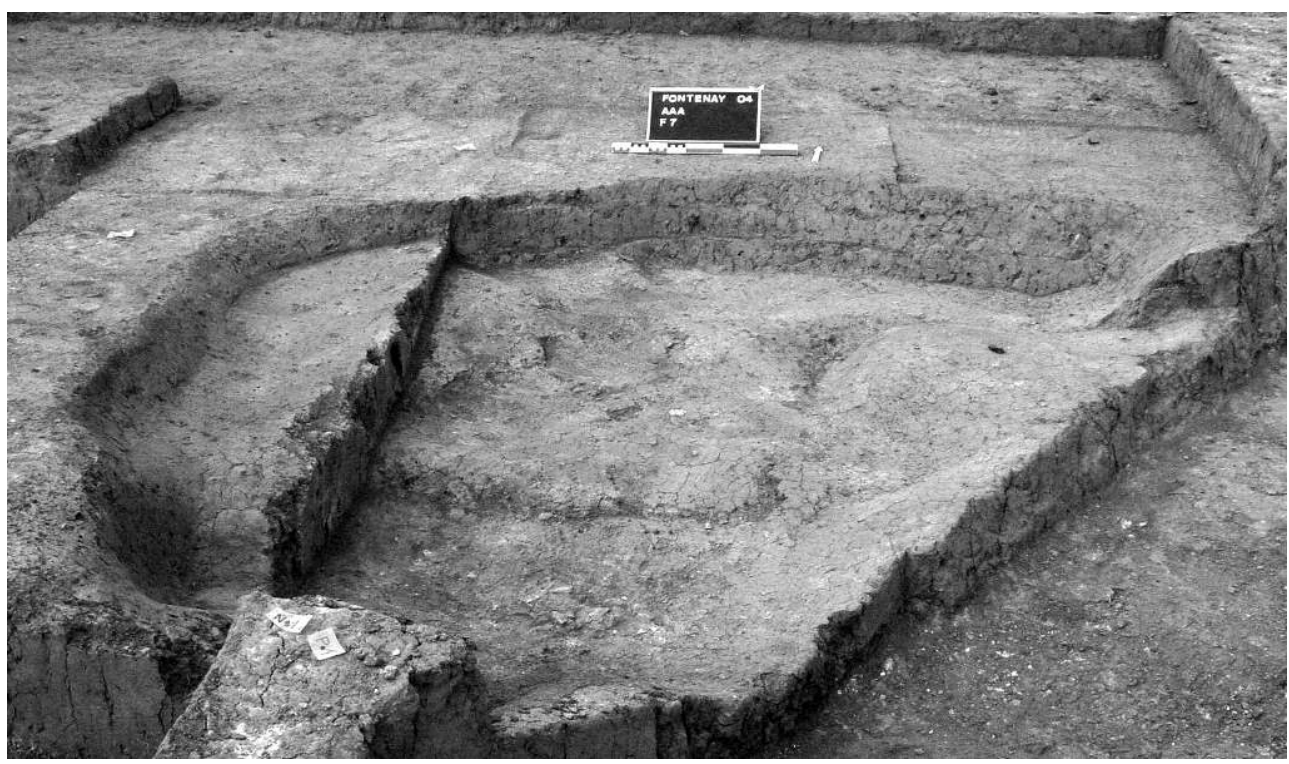

\section{Les fosses sud (F6 et F25)}

11 La fosse 6 a été repérée lors du premier décapage, dix à quinze centimètres plus haut que la fosse nord, grâce à la présence de plusieurs concentrations de mobilier (fig. 5). La lecture des limites de cette structure s'est avérée très difficile, à l'exception de son extrémité ouest ou les rejets domestiques sont abondants. La présence de microcharbons et de quelques artefacts au sein de son remplissage limoneux nous a permis de tenter de restituer la forme de cette fosse. Il s'agit d'une excavation oblongue de près de dix-sept mètres de longueur, au profil évasé. Son fond a été difficile à distinguer du sédiment en place: quelques coupes montrent qu'il est irrégulier et pourrait comprendre quelques lobes qui atteignent le substrat calcaire. La profondeur maximum de cette fosse, estimée essentiellement par la présence de mobilier, ne dépasse pas les quarante centimètres. La coupe réalisée vers l'extrémité ouest de cette fosse, au niveau de la plus importante concentration de mobilier semble révéler la présence de deux remplissages distincts : le premier, riche en artefacts, est assez charbonneux, et un deuxième, a priori stérile, qui est composé d'un limon roux. 
Figure 5 : Vue d'une coupe de la fosse sud (fosse 6). Figure 5: View of a section of the southern pit (pit 6).

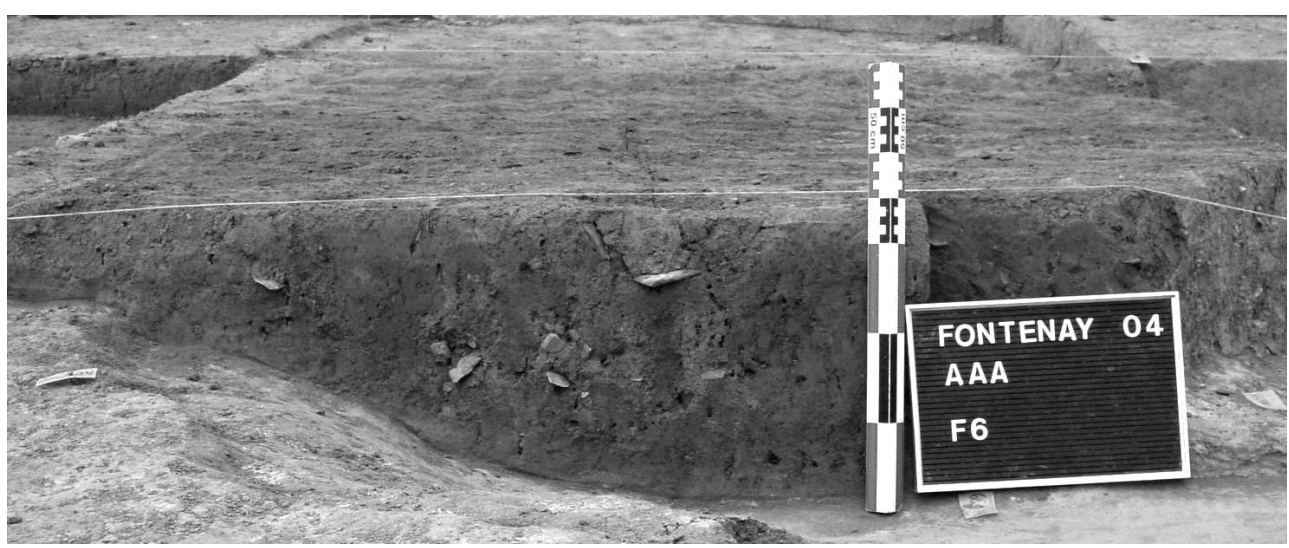

La fosse 25 a été localisée lors de la fouille. Sa partie supérieure est tronquée par des structures de périodes postérieures. Il s'agit d'une grande fosse, à peu près circulaire de 4,5 mètres de diamètre au profil évasé (fig. 6). Il semble que cette structure coupe le substrat calcaire sur une dizaine de centimètres. Deux remplissages se distinguent : le premier, un limon argileux gris, comble la partie supérieure de l'excavation. Il comprend de nombreux micro-charbons et des nodules de terre rubéfiée et est assez riche en artefacts. Le second, très peu « anthropisé » correspond à une couche de limon argileux rougeâtre, difficile à distinguer du sédiment en place.

Figure 6 : Coupe de la fosse sud (fosse 25).

Figure 6: Section of the southern pit (pit 25).

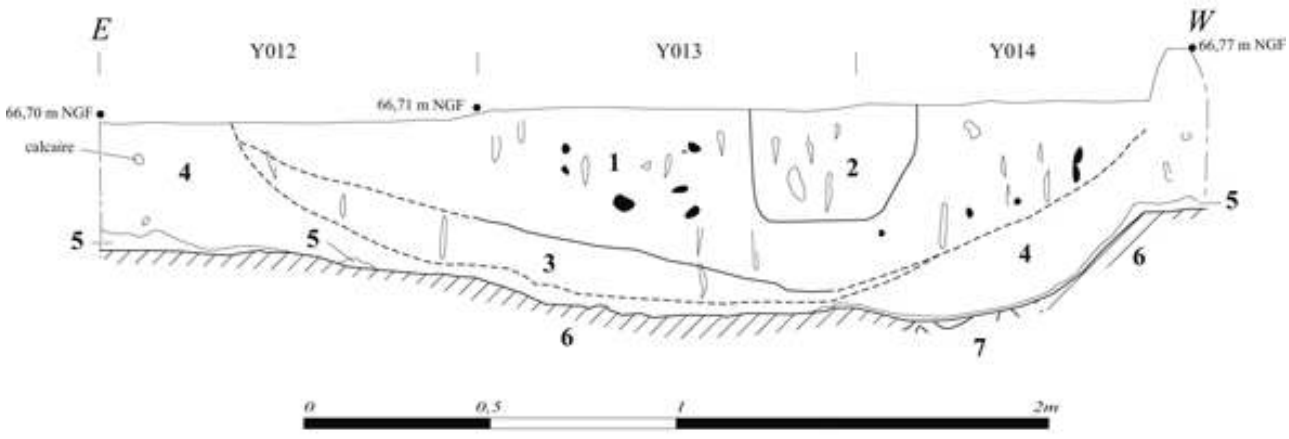

1- fosse : remplissage gris comprenant de nombreux

éléments de mobilier

2- fossé F27 : remplissage limoneux de couleur gris clair

3- limon gris-brun

4- limon brun-rouge

5- limon brun calcaire

mobilier néolithique

6- substrat : calcaire poudreux

7- calcaire noduleux chenaux de lombrics

\section{Analyse micromorphologique par Cécile Germain}

Ces fosses sud ont fait l'objet d'une analyse micromorphologique. L'objectif étant d'identifier la nature de leur remplissage afin d'expliquer la difficulté à l'identifier de leur encaissant. Cette analyse vise également à répondre aux questionnements des archéologues quant au "véritable" fond des structures lorsqu'elles excavent le substrat : est-ce lié à la décarbonatation post-néolithique qui s'est effectuée aux dépens 
du substrat calcaire poudreux comme cela a été observé sur le site du Néolithique ancien de Colombelles? Ou bien s'agit-il d'un creusement anthropique intentionnel du substrat? Pour une meilleure interprétation des résultats de ces analyses, ceux-ci ont été comparés aux résultats de l'analyse des formations du site en dehors des structures archéologiques...

Deux prélèvements en blocs non perturbés effectués dans le remplissage de cette fosse ont été analysés en lame mince. L'échantillon 6 (carré AA10, fosse 6) vise à caractériser la couche rouge observée dans le comblement inférieur de cette fosse, alors que l'échantillon 10 (carré AB10 fosse 6) a pour objectif d'identifier un niveau grisâtre plus ou moins foncé parfois observé juste au-dessus du substrat calcaire et sous le niveau rouge évoqué précédemment.

\section{L'échantillon 6, description micromorphologique}

En lame mince, la «couche rouge » est composée d'un sédiment à microstructure compacte à polyédrique, de texture limoneuse à limono-argileuse. Les limons sont des quartzs d'origine lœssique. Quelques esquilles de silex, dont une de forme presque carrée et anguleuse entrent également dans la composition de ce sédiment. Elles pourraient être d'origine anthropique. En outre, de nombreuses particules de matière organique, souvent de section égale ou légèrement supérieure à deux cents micromètres, ont été également identifiées. L'observation de structures cellulaires de certaines d'entres elles suggère une origine végétale. Dans son ensemble, ce sédiment est décarbonaté. Cependant, des bioturbations probablement liées aux vers de terre géophages (organisations excrémentales en nid d'abeille et en pile d'assiette ; Fedoroff et Courty, 1994) présentent quelques grains carbonatés. Enfin, ce sédiment est caractérisé par de nombreux traits texturaux qui se développent sous la forme de revêtements argileux orangés. Ces traits pédologiques sont caractéristiques d'un horizon illuvial Bt. La plupart tapisse les vides du sédiment sauf dans les zones bioturbées où seulement des papules (fragments de ces revêtements argileux) peuvent être observées. Certaines des particules organiques sont «enrobées» par des revêtements argileux.

\section{L'échantillon 10, description micromorphologique}

16 Aux échelles microscopiques, cet échantillon est caractérisé par une microstructure grumeleuse bien développée. Celle-ci révèle un sédiment très bioturbé. Cette importante bioturbation est également exprimée par des zones présentant une organisation excrémentale en nids d'abeilles ou en piles d'assiettes liées aux vers de terre géophages. Par ailleurs, ce sédiment est de texture limoneuse avec des limons quartzeux d'origine probablement lœssique. Quelques grains sableux carbonatés ont été observés mais dans l'ensemble, il s'agit d'un sédiment décarbonaté. Des particules organiques, moins grosses et peut-être moins nombreuses que l'échantillon 6 , entrent aussi dans la constitution de ce niveau. Enfin, des traits texturaux argileux orangés ont également été observés. Ils sont en quantité moins importante que dans l'échantillon 6 . Il s'agit essentiellement de papules. Quelques revêtements argileux en position de vide s'observent vers la moitié inférieure de la lame ou par le biais d'agrégats de Bt dispersés dans la lame mince... 


\section{Interprétation}

17 S'agissant de l'échantillon 6, la quantité importante de particules organiques observées dans cet échantillon suggère un sédiment d'origine probablement anthropique. Ce sédiment a connu une évolution pédologique post-dépositionnelle importante comme en témoigne l'enrobage de quelques-unes de ces particules par des revêtements argileux. Par ailleurs, le fait que ces revêtements se retrouvent en position de vide suggère également leur développement in situ et donc, une évolution pédologique de type luvisol postérieurement à l'occupation néolithique. Cette évolution pédologique atteste de l'abandon du site pendant un temps long permettant le développement d'une couverture végétale continue et durable et la reprise de la pédogenèse avec notamment, l'illuviation des argiles. Cette longue évolution pédologique post-abandon explique également les difficultés de lecture des structures archéologiques. Enfin, ce sédiment est assez fortement bioturbé. Ces bioturbations peuvent être récentes. En effet, elles sont du même type que celles observées dans le profil pédologique analysé hors structure. Celles-ci tendent à re-carbonater l'horizon Bt conservé.

Quant à l'échantillon 10, correspondant à un niveau brun-gris, retrouvé parfois à la base du comblement sous le niveau rouge (échantillon 6), sa très forte bioturbation limite son interprétation. Néanmoins, sa position stratigraphique sous-jacente au niveau rouge semble indiquer que cette microstructure grumeleuse pourrait être antérieure au Néolithique et donc héritée. Par ailleurs, les papules et les agrégats issus de l'horizon illuvial Bt suggèrent que ce sédiment est une colluvion. Si tel est le cas, ce niveau retrouvé à la base du comblement de la fosse 6 pourrait être une colluvion qui remobilise à la fois du sédiment issu du Bt et peut-être d'un horizon pédologique $\mathrm{A}$ de surface, d'où cette microstructure grumeleuse bien développée. En tout cas, d'après ses caractéristiques, ce niveau ne semble pas lié à une évolution post-abandon du site comme la décarbonatation... Elle serait accompagnée d'illuviation.

\section{Les vestiges du bâtiment d'habitation}

19 À la suite du premier décapage, aucune trace probante de trou de poteau ne s'est révélée, à l'exception peut-être d'un fait (F10) qui est assez riche en charbon de bois et comprend quelques silex. Les autres traces repérées pourraient correspondre à des poches de sédiments légèrement plus grises, visibles épisodiquement, probablement dues à des irrégularités du décapage, à des traces de culture ou encore à des bioturbations. Mais c'est à l'occasion du deuxième décapage, dix à vingt centimètres plus bas que sont apparues quelques excavations dans la zone centrale correspondant à l'emplacement présumé de la maison (fig. 7). La plus importante est F35. Elle est circulaire et mesure 1,20 mètre de diamètre. Son profil est en cuvette et son remplissage central de couleur grise comprend de nombreux micro-charbons, des morceaux de terre rubéfiée (torchis) et quelques artefacts en silex. Sa profondeur est d'environ trente centimètres et ne semble pas atteindre le substrat. Cependant, le décapage au niveau du calcaire révèle à cet emplacement une excavation plus large qui paraît difficilement être une coïncidence. Ce qui signifierait que la partie fouillée, s'il s'agit d'un trou de poteau, correspond seulement au fantôme de la pièce en bois où à l'empreinte comblée de cette dernière dans le cas où le poteau aurait été démonté. 


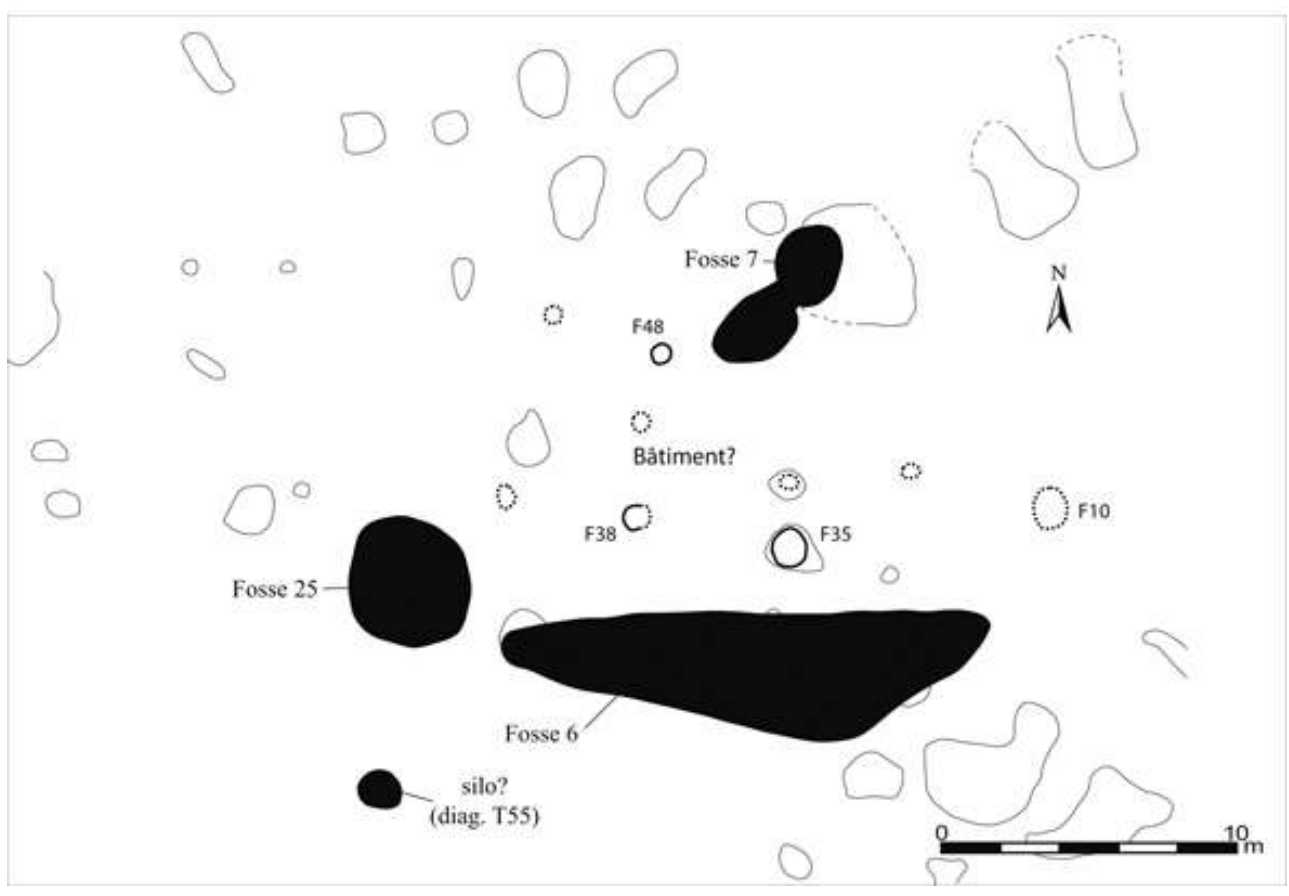

Deux autres structures (F38 et F48) de taille plus modeste (environ $70 \mathrm{~cm}$ de diamètre) aux limites à peu près lisibles possèdent un remplissage similaire incluant des éléments de torchis. D'autres traces, plus succinctes, caractérisées par des concentrations de micro-charbons ont pu être relevées. Trois dépressions dans le substrat témoignent peut-être également de la présence de trous de poteaux. Le plan de ces différentes "structures" semble fonctionner assez bien avec une organisation en tierce d'un bâtiment de type danubien.

\section{3. Étude de la répartition du mobilier}

\section{Présentation}

Le mobilier mis au jour a été localisé à une précision de moins de un quart de mètre carré et par passes, dit US, de dix centimètres. Il faut toutefois signaler la différence de hauteur de décapage de dix à quinze centimètres au niveau de la fosse nord, due à l'ouverture d'une fenêtre d'évaluation lors du diagnostic. Il y a donc un décalage dans l'enregistrement, de près d'une «US » entre la fosse nord et les fosses sud.

Les premières informations livrées par cette étude concernent la localisation des structures et leur morphologie (forme, profondeur...). Les limites du lobe sud de la fosse 7 n'ont pas pu être reconnues sur le terrain. La position des artefacts nous permet de proposer une restitution en plan de la structure. La présence de mobilier enregistré en US 5 dans les fosses 25 et $\mathrm{F} 7$ (sud) implique une profondeur supérieure à quarante centimètres des deux excavations.

Le deuxième décapage n'a pratiquement pas livré d'artefacts, à l'exception de la structure F35 dont le mobilier a été recalé en US 3. 
24 Les éléments pris en compte pour cette étude sont le matériel lithique en silex, la céramique et les fragments de torchis, en raison de leur «origine " anthropique indéniable.

On observe du mobilier en dehors des limites identifiées des structures pour les US 1 et 2 , principalement au nord de la fosse 6. Il s'agit essentiellement de petites pièces en silex; les quelques tessons de poteries trouvés en dehors des fosses sont de très petite taille, extrêmement roulés et de toutes périodes (gallo-romaine, protohistorique...). Cet éparpillement des artefacts dans les quinze premiers centimètres est la conséquence de remaniements du sédiment par des labours, des animaux fouisseurs ou autres creusements anthropiques.

\section{Proportion de mobilier entre les fosses sud et la fosse nord}

Les fosses sud ont livré $82 \%$ des pièces en silex mises au jour, dont $40 \%$ proviennent de l'extrémité ouest de la fosse 6 (fig. 8, fig. 9). On note un équilibre dans la proportion de tessons de poterie entre les fosses sud et la fosse nord, avec 49,5\% de céramique issue de F7. Si l'on tient compte des tessons trouvés pendant le diagnostic, la tendance s'inverse car les deux tiers de la céramique découverte alors proviennent de la fosse nord. On remarque également, comme pour le matériel en silex, que l'essentiel des tessons de poteries découverts dans F6 est issu de la concentration de mobilier située à son extrémité ouest, soit $26,5 \%$ du total.

27 Nous constatons que la majorité du mobilier provient des fosses sud, essentiellement d'une petite portion de $\mathrm{F} 6$ d'environ un mètre carré où a été trouvé plus de $60 \% \mathrm{du}$ matériel lithique en silex et plus de $80 \%$ des céramiques de cette structure. La proportion de mobilier découvert dans les deux lobes de la fosse nord est plus équilibrée avec un avantage pour le lobe sud notamment en US 1. 
Figure 8 : Plan de répartition du mobilier en silex. Figure 8: Spatial distribution of lithic artefacts.

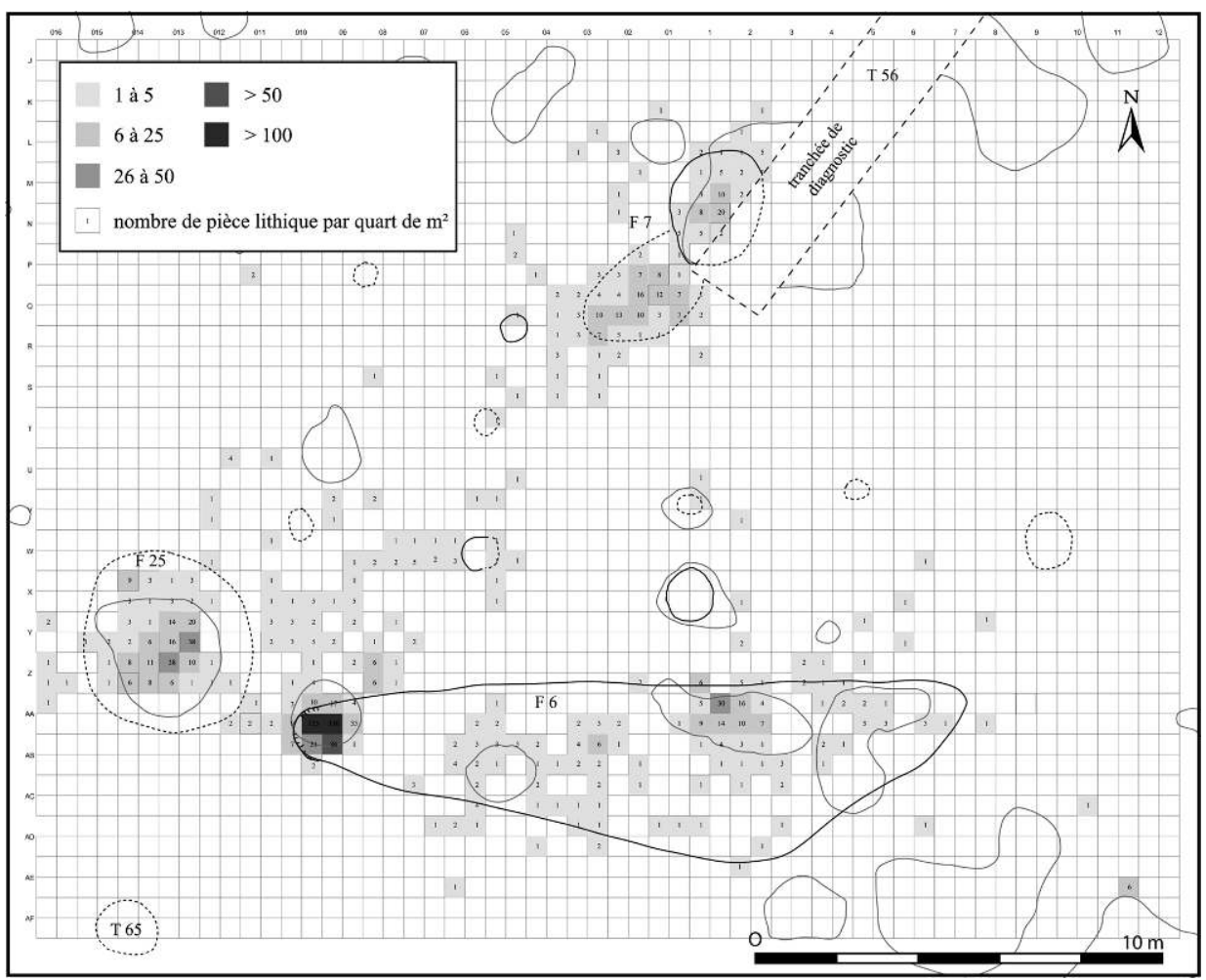

Figure 9 : Plan de répartition du mobilier céramique. Figure 9: Spatial distribution of ceramic artefacts.

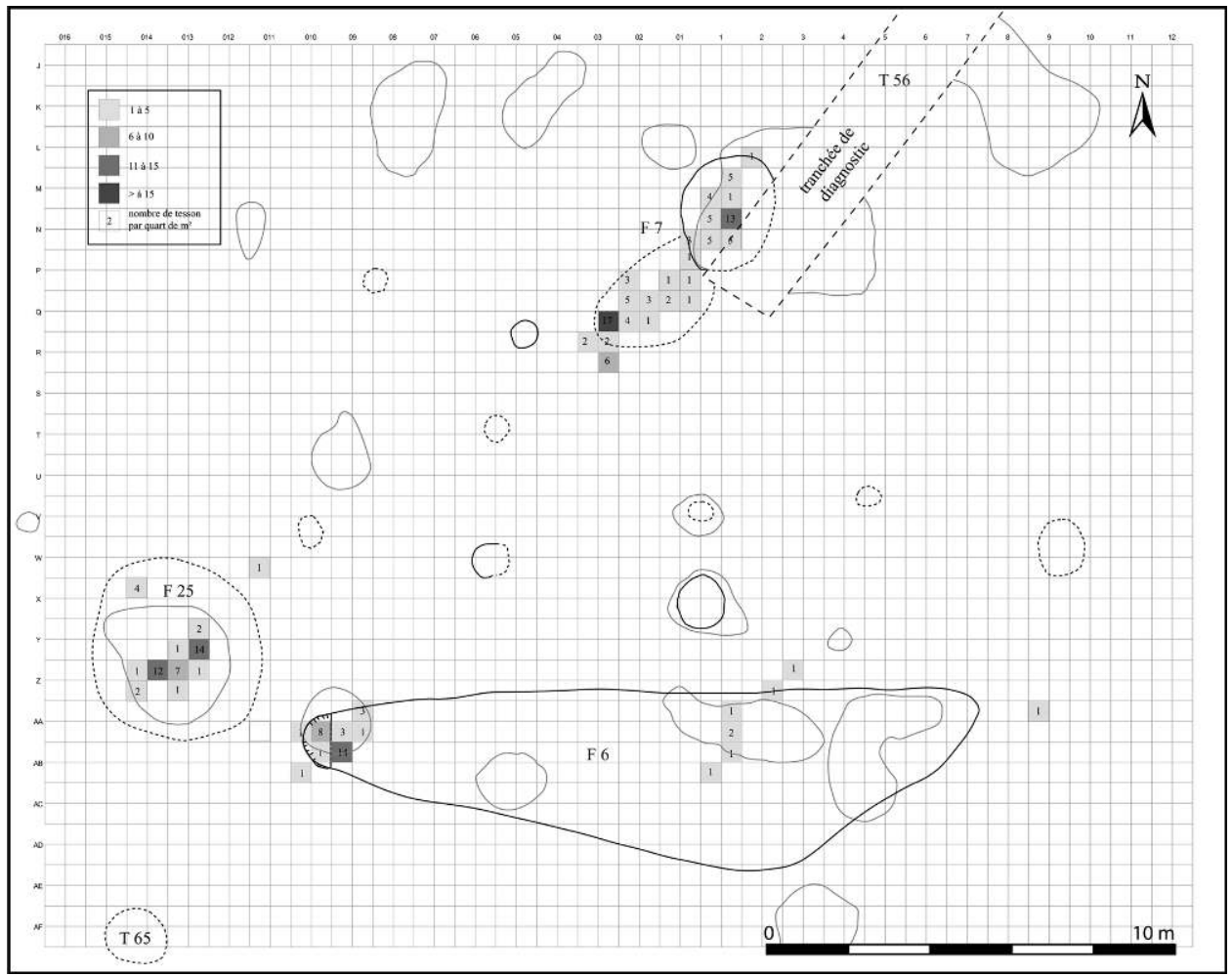


Il est assez fréquent que les rejets se présentent sous forme de concentrations dans les fosses latérales de maisons de type danubien. C'est le cas pour plusieurs maisons de Poses «Sur La Mare» (Bostyn et al., 2003) et pour celle de Mondeville « le Haut SaintMartin » (Marcigny et al., 2010, p. 132-133). La plus importante des concentrations est située, le plus souvent, dans la fosse sud au droit de la pièce précédent le couloir principal. Les autres concentrations de mobilier, dont deux dans F6, pourraient indiquer l'emplacement d'ouvertures dans le bâtiment.

29 La fosse nord, relativement riche en mobilier, est au même niveau qu'une des concentrations de F6 donc, peut-être à proximité d'une ouverture symétrique à celle donnant sur la fosse sud.

Sur le site de Poses "Sur La Mare", le couloir principal de plusieurs des maisons débouche, au sud, sur une interruption de la fosse latérale; les plus importants rejets domestiques sont situés dans la fosse à l'est (Bostyn et al., 2003). Ce schéma semble s'appliquer à Fontenay-le-Marmion, le couloir principal du bâtiment pourrait être situé face à l'espace entre F6 et F25.

\section{Proportion de mobilier par US}

\section{Structure F6}

On compte, dans cette structure, un maximum de quatre niveaux de dix centimètres comprenant du mobilier. La grande majorité du matériel céramique ou lithique provient des vingt premiers centimètres du remplissage de F6 (fig. 10). Il existe un écart important entre US 2 et US 3 puisqu'il est supérieur à $200 \%$, celui entre US 3 et US 4 est comparable. L'aspect du graphe de proportion du mobilier en céramique par US est similaire à celui du matériel lithique en silex; la seule différence notable est que l'on trouve plus de lithique dans la première passe alors que la quantité de tessons de poteries est maximale en US 2 . 
Figure 10 : Graphiques de proportion de silex (A) et de céramique (B) dans les fosses par unité stratigraphique.

Figure 10: Graphs representing the proportion of pottery and flint in the pits by stratigraphic unit.
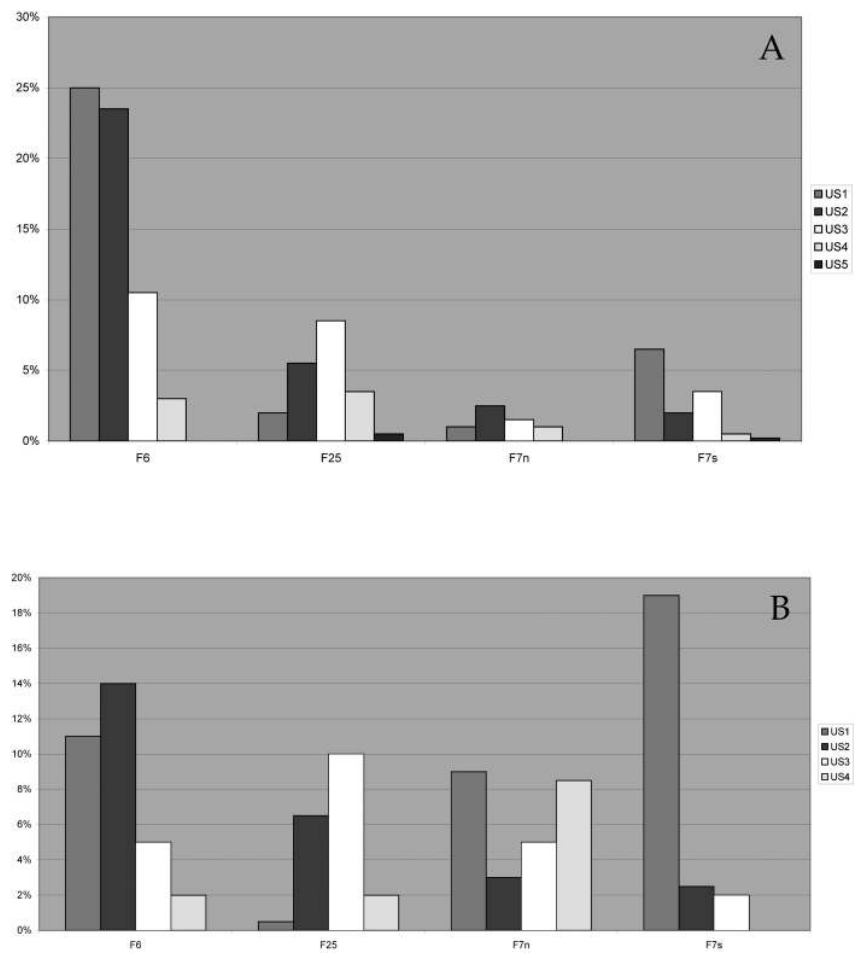

\section{Structure F25}

Dans cette fosse, on trouve du mobilier dans cinq US. Contrairement au graphe de F6, l'US 1 est presque stérile et la quantité de mobilier croît pour les trois premiers niveaux ; l'US 3 a livré la plus grande part de mobilier céramique et lithique (fig. 10). La proportion d'artefact décroît rapidement pour les deux derniers niveaux.

\section{Structure F7}

On compte cinq passes contenant du mobilier dans le lobe sud et quatre dans le nord, en excluant les dix à vingt centimètres de remplissage décapés lors du diagnostic archéologique. Globalement, le mobilier a tendance à décroître vers le fond, sauf pour la céramique du lobe nord dont la proportion augmente à partir de l'US 2 (fig. 10).

\section{Interprétation}

En règle générale, on constate que la part de mobilier décroit vers le fond de la structure. Il est fréquent que les fosses attenantes des maisons de la culture VSG livrent l'essentiel de leurs artefacts dans le remplissage supérieur ou médian; le premier niveau (inférieur) est souvent stérile (Bostyn et al., 2003 ; Chancerel et al., 2006). Les anomalies à ce schéma relevées dans certaines fosses de ce site ont une cause logique : la pauvreté en mobilier en haut du remplissage de F25 s'explique par son recoupement avec un fossé protohistorique et une chaussée creusée ; la forte densité de fragments de 
céramiques au fond du lobe nord est principalement due à la présence de nombreux fragments d'une ou de deux poteries.

Compte tenu de l'arasement du site de Fontenay-le-Marmion, tronqué sur une épaisseur de plus de quarante centimètres par l'érosion agricole, on peut estimer que certaines fosses devaient atteindre près de un mètre de profondeur (F25 et F7).

Les couches riches en rejets domestiques semblent localisées sur un niveau de stabilisation (colluvionnement rapide), elles sont les plus affectées par la mise en culture. La proportion de mobilier conservé sur ce site est, en conséquence, loin d'être représentative de l'activité domestique de l'occupation. La céramique est très altérée en surface des fosses, contrairement aux pièces lithiques que l'on retrouve éparses jusqu'au sommet des labours.

\section{Bilan de l'étude de répartition du mobilier}

37 Ce site d'habitat du Néolithique ancien est assez arasé, ce qui explique la relative faiblesse de la quantité d'artefacts mis au jour et notamment, celle du mobilier le plus fragile, la céramique. Les informations livrées par l'étude de la répartition spatiale du mobilier issu des fosses latérales correspondent à un schéma courant pour les sites de ce type.

38 - Les fosses sud comprennent plus de mobilier que la fosse nord F7 contient autant de fragments de poterie que F6 et F25, mais cette fosse est plus profonde et moins arasée.

39 - On retrouve des pics de densité de matériel dans les fosses probablement à proximité d'ouvertures dans la maison. La plus importante des concentrations est fréquemment localisée au droit du couloir de la maison. Ce couloir pourrait être situé face à l'espace entre F6 et F25.

40 - Le mode de remplissage des fosses attenantes est également classique avec un premier comblement naturel sans doute rapide (colluvionnement) puis un second comprenant par endroit des rejets domestiques. Il est probable que les déchets soient rejetés «massivement » dans les fosses, comme en témoigne la présence de fragments de poterie de même individu dans plusieurs US. Il semble que l'essentiel des artefacts découverts dans les fosses correspondent à des rejets indirects. On retrouve également des tessons d'une même pièce dans plusieurs fosses. Ces rejets dans les fosses attenantes pourraient être consécutifs à un nettoyage à l'intérieur de la maison?

\section{La céramique}

\section{Étude quantitative et qualitative}

41 L'ensemble céramique issu des différentes fosses latérales est relativement pauvre. Seuls 261 tessons de poterie (pour un poids de 1,094 kg) ont été mis au jour. Il faut ajouter les cinquante fragments $(430 \mathrm{~g})$ trouvés lors du diagnostic dans ces mêmes fosses. Pour comparaison, la plupart des sites de cette période livrent plus de sept kilos de poteries par unité d'habitation. Les maisons «VSG» de Poses comprennent entre six et vingt-deux kilos de céramiques (Lanchon in Bostyn et al., 2003).

Ici, comme pour le site de Mondeville « le Haut Saint-Martin » (125 tessons seulement), l'important arasement du site est la première cause de la faible quantité de fragments 
de poteries conservés. L'étude de la répartition spatiale du mobilier montre que le sommet du remplissage des fosses sud est l'endroit le plus riche en artefacts et est également celui le plus affecté par l'érosion.

L'état de conservation des fragments de poteries est globalement assez mauvais et les tessons de grandes tailles sont plutôt rares. On observe que certains morceaux sont en très bon état alors que d'autres sont très érodés, leurs surfaces externe et/ou interne sont très émoussées. Ces différences de conservation sont constatées sur des fragments appartenant au même récipient.

Nous avons identifié très peu de formes céramiques, puisque seuls cinq bords, une anse et deux éléments de décor (un petit tesson avec un décor d'incision et des petits boutons jumelés) sont présents dans la collection (fig. 11). L'unique pièce restituable est un «bol » à forme dite "en bombe » comprenant au moins deux paires de très petits boutons jumelés, situés sur sa panse, au niveau de son diamètre maximal (fig. 11, no 4). Nous comptons également deux cols de bouteilles, dont une de grande taille (diamètre = $17 \mathrm{~cm}$ ) (fig. 11, no 2 et 5). Les deux autres bords pourraient appartenir à des vases de forme à profil en « $\mathrm{U}$ » (fig. 11, no 1 et 3). Un très petit fragment de poterie décoré d'incisions (en arête de poisson ?), découvert lors du diagnostic archéologique, provient de la fosse nord (fig. 11 no 7).

Figure 11 : Planche céramique. Dessins A. Cocollos.

Figure 11: Pottery. Drawings A. Cocollos.

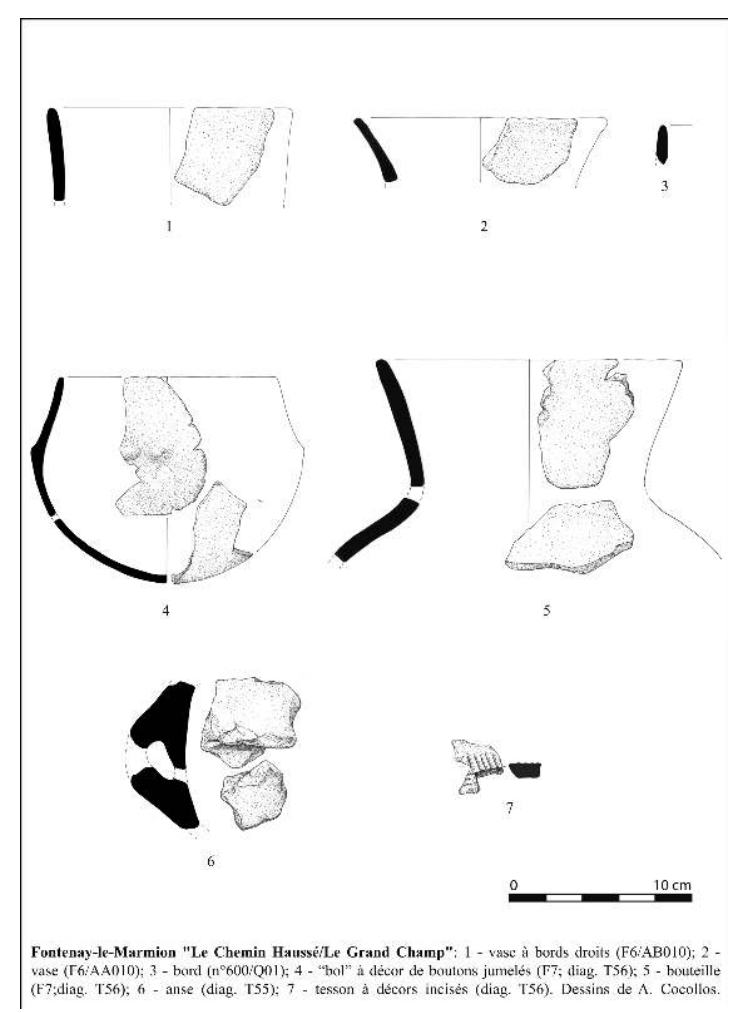

Nous avons classé cet ensemble céramique en treize groupes aux caractéristiques différentes (aspect de la pâte, inclusions, épaisseurs des parois...; tabl. 1). Ces groupes correspondent au nombre probable d'individus céramiques répertoriés sur ce site. On observe, dans la plupart des groupes, des provenances dans les fosses sud et nord. Il est 
même évident que certains tessons du même vase ont été trouvés dans les deux fosses F6 et F7 ; c'est le cas pour des fragments de la grande bouteille.

Tableau 1 : Description des groupes des céramiques. Table 1: Description of the pottery groups.

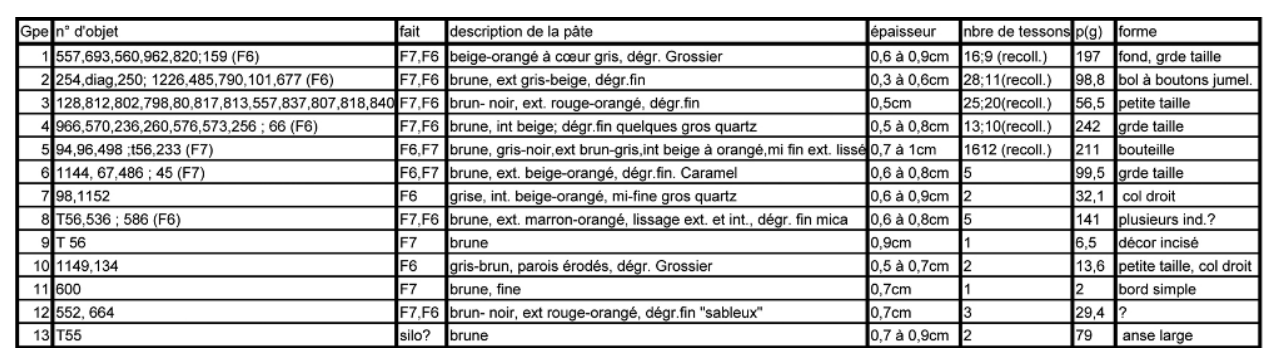

\section{Étude pétrographique des pâtes céramiques par Xavier Savary et Denis Jan}

Les pâtes des treize groupes ont été observées à l'œil nu puis à la loupe binoculaire. Au premier examen, les pâtes sont apparues assez hétérogènes puisque, en raison de la finesse des constituants, l'observation s'est basée essentiellement sur la présence/ absence d'éléments noirâtres, jaunâtres ou orangés et sur la proportion de quartzs grossiers, somme toute assez faible sur tous les vases. Le second examen a permis de mettre en évidence un aspect général finement sableux commun à la majorité des pâtes et qui venait alors reléguer au second plan les critères retenus auparavant. Au bilan, dix des treize groupes apparaissaient assez constants dans leur composition puisqu'ils affichaient presque systématiquement une matrice argileuse assez fine, envahie par un sable fin à moyen, dont la majorité des grains s'apparentait à des grains de quartz souvent luisants, dont la taille pouvait régulièrement atteindre le millimètre pour un diamètre maximum de deux millimètres. Les variations à l'intérieur de cette grande catégorie reposaient alors sur la couleur de la pâte et sur la présence/absence et au cas échéant, la proportion des éléments jaunâtres, orangés et/ou noirâtres. Une première analyse microscopique à valeur de test a été entreprise afin de confirmer la légitimité de cette grande catégorie, mais aussi et surtout, pour mieux caractériser ces pâtes ainsi que celles apparues plus marginales à l'examen macroscopique.

La grande majorité des pâtes contient donc une proportion importante de particules sableuses dominées par des grains de quartz plutôt anguleux. Ces derniers sont accompagnés de feldspaths dominés par l'orthose et de rares micas (biotite et muscovite) disséminés dans la matrice argileuse. Quelques tourmalines appartiennent accessoirement à ce cortège minéralogique. D'autres grains, visibles à l'œil nu, sont omniprésents dans la majorité des pâtes. Il s'agit d'éléments à matrice argileuse souvent ferruginisés ou carbonisés, dont la couleur tranche avec le reste de la pâte mais dont le faciès est le plus souvent assez proche de celle-ci. Dans certains échantillons, ces derniers éléments sont accompagnés d'autres grains de ce type mais dont le faciès apparaît plus dense et les éléments, les quartzs notamment, plus fréquents. L'interprétation de ces grains n'est pas sans poser problème même si dans certains cas, à l'œil nu comme au microscope, ils semblent pouvoir être facilement assimilés à de la chamotte. Pourtant, il pourrait également s'agir de grumeaux d'argile issus d'un mauvais malaxage ou d'une évolution pédo-génétique des argiles originelles. 

carbonisées dans deux pâtes à particules sableuses et dans une pâte à éléments d'origine magmatique (Jan, 2010). Dans une pâte à particules sableuses, les inclusions végétales, peu abondantes, se révèlent sous la forme de vides rectilignes et circulaires contenant de la matière carbonisée (fig. 12). Dans les deux autres pâtes, les restes végétaux sont en quantité très abondante. La pâte à particules sableuses contient de nombreuses empreintes végétales qui se révèlent sous la forme de vides ovales et circulaires contenant de la matière végétale carbonisée, dont la structure cellulaire est parfois visible et également, de vides rectilignes très fins. La pâte à éléments d'origine magmatique renferme une forte quantité d'inclusions végétales qui prennent la forme de vides circulaires et oblongs, de vides rectilignes fins, de vides rectilignes épais contenant de la matière végétale carbonisée et de vides présentant une morphologie végétale. L'identification de ces empreintes végétales est complexe mais l'examen macroscopique et microscopique des pâtes, couplé à l'élaboration d'un protocole expérimental, permet de se focaliser sur un groupe de plantes particulier, celui des Bryophytes. Il est très probable que ces inclusions végétales appartiennent à la classe des mousses (Jan, 2011). 
Figure 12 : Exemple d'empreintes végétales dans les pâtes céramiques, en lumière polarisée non analysée.

Figure 12: Example of vegetal prints in the pottery fabric, photographed in polarized light (non analysed).

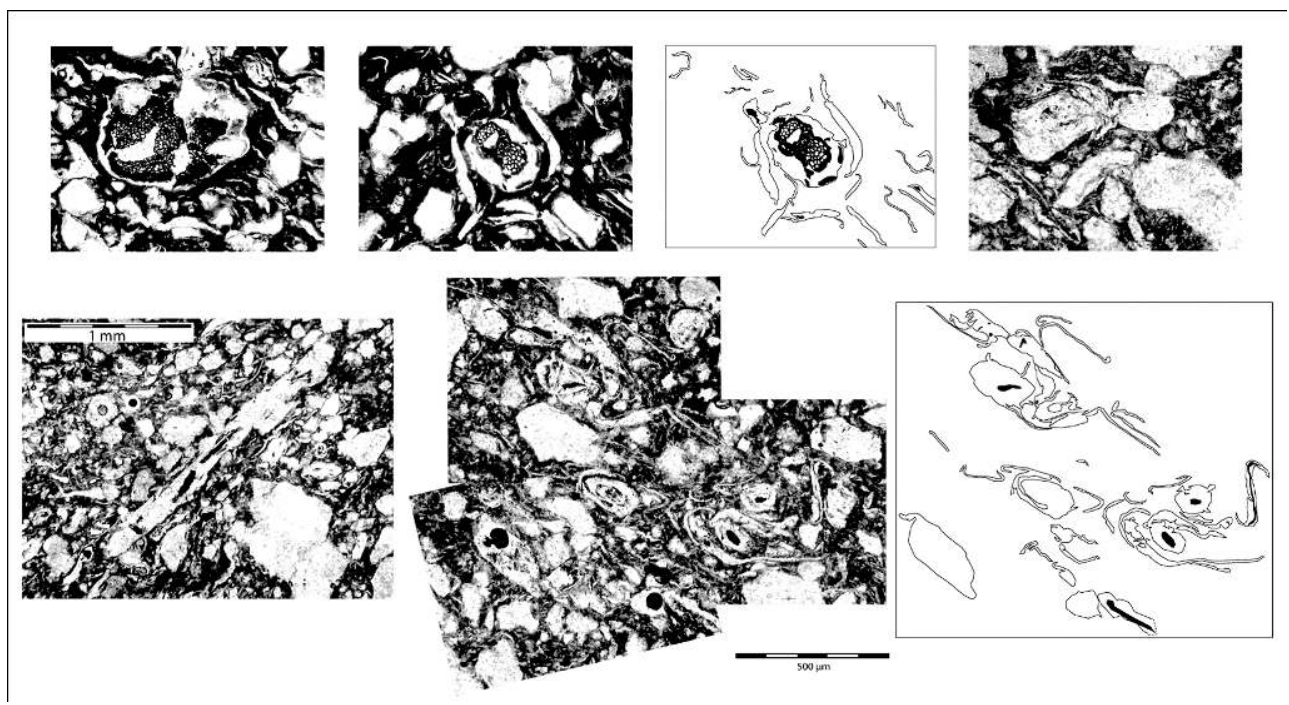

\section{Bilan de l'étude céramique}

Ce petit ensemble au nombre d'individus très restreint permet toutefois d'apporter quelques informations.

52 - Le taux d'usure différent sur des tessons d'une même pièce montre que certains fragments ont été exposés aux intempéries, avant d'être rejetés dans les fosses. De même, comme le démontre l'étude de la répartition spatiale, on trouve des éléments de mêmes vases à différentes profondeurs. Il est donc probable que certains rejets domestiques soient déposés, dans un deuxième temps, dans les fosses latérales de la maison. Il semble même que ce comblement, peut-être partiel des fosses, soit assez rapide.

53 - La question de l'appartenance chronologique est bien définie, malgré un nombre réduit de formes, grâce à des éléments plutôt bien caractérisés. Les très grandes bouteilles comprenant un diamètre à l'ouverture supérieur à quinze centimètres se retrouvent souvent dans les corpus céramiques de la culture VSG, d'après Yves Lanchon (Bostyn et al., 2003, p. 87). Les vases à petits boutons jumelés sont également fréquents sur les sites de la phase ancienne et moyenne VSG, tout comme les décors incisés en arrête de poisson.

\section{L'industrie lithique par Laurent Juhel avec les contributions de Jean-Paul Caspar ( ()}

\section{Origine du mobilier lithique}

54 Le mobilier est issu de deux ensembles interprétés en tant que "fosse nord " (F7) et «fosse sud » (F6). À l'extrémité ouest de F6, une concentration de produits lithiques correspond au principal rejet dans cette structure. Dans le prolongement de F6, vers l'ouest, le fait F25 a lui aussi livré du mobilier néolithique ancien. Ces éléments ont été 
intégrés au décompte de la fosse sud. Un troisième ensemble est constitué des pièces découvertes en dehors des faits identifiés, mais sur l'emprise probable de l'habitat. Leur aspect physique ainsi que leur typologie ne laissent planer guère de doute sur leur appartenance au même ensemble que les structures excavées.

On remarque un net déséquilibre, tant en nombre qu'en poids, entre les ensembles interprétés comme « fosse nord » et « fosse sud » (tab. 2).

Tableau 2 : Répartition du mobilier lithique.

Table 2: Spatial distribution of lithic artefacts.

\begin{tabular}{|c|c|c|c|}
\cline { 2 - 4 } \multicolumn{1}{c|}{} & «fosse nord » & «fosse sud » & « hors fosses » \\
\hline faits & F7 + T56 & F6 + F25 + T55 & - \\
\hline nombre d'objets & 160 & 802 & 55 \\
\hline poids & 2461 & 10850 & 1348 \\
\hline
\end{tabular}

\section{Les matières premières}

Un silex jurassique à grain fin de la plaine de Caen, communément nommé silex « du Cinglais » représente $96 \%$ de la série. Au sein de la série étudiée, ce matériau présente des variations de couleur dont la perception est complexifiée par un léger voile de patine à dominante grise, non systématique, mais souvent accompagnée de vermicules ou de taches blanchâtres. Une grande partie du débitage a, selon toute vraisemblance, été réalisée dans un silex initialement brun foncé, correspondant au faciès classiquement connu pour ce matériau, mais certains éclats présentent également des zonages sous-corticaux passant du gris clair au beige, voire au jaune. Ce silex présente un grain très fin, propice à un débitage laminaire de qualité, mais une minorité d'artefacts témoigne de l'exploitation de blocs plus ou moins désilicifiés, tels qu'ils peuvent se présenter dans les couches supérieures des argiles (Coutard, 1998).

Un silex jaune orangé représente $4 \%$ de la série. Une disponibilité de ce matériau au niveau régional (pays d'Auge ?) peut être envisagée. Seulement un ou deux blocs de taille réduite ont été exploités, sans traitement spécifique.

Une silicification tertiaire est représentée par un objet, un fragment de lame à crête esquillée (fig. 18, no 4). Il s'agit d'une opalite, dont les affleurements les plus proches sont à rechercher dans les Yvelines, à cent cinquante kilomètres du site, ou encore dans le Val-de-Loire (Blanchard et Forré, 2003).

Un burin sur lame est réalisé dans un silex cénomanien noir (fig. 14, no 11). Ce silex crétacé n'est pas strictement local, mais une origine littorale proche d'une trentaine de kilomètres au nord du site est probable.

60 Un quartzite blanc est représenté par une petite herminette polie, ainsi qu'un éclat issu de ce même objet (fig. 18, no 1 et 2). Au niveau régional, ce type de matériau peut être rencontré sur les franges du Massif armoricain, sans plus de précision. L'absence de surfaces naturelles sur cet objet taillé et poli ne permet pas de définir son contexte de collecte. Il peut provenir d'un affleurement en place, d'un épandage triastique, ou encore d'une nappe alluviale. 


\section{Caractérisation des productions}

61 Deux types de production sur silex, lame et éclat, se côtoient à Fontenay-le-Marmion, comme cela a largement été mis en évidence pour les assemblages du Néolithique ancien (Bostyn, 1994). Cependant, la production laminaire s'avère largement prépondérante ( $45 \%$ du total des produits, outils inclus) sur la production d'éclats véritables $(12,5 \%)$. Le reste de la série est constitué par la catégorie cassons et débris (33,5\%), et par des éléments indéterminés (9\%).

\section{La production laminaire} débitage :

- un fragment proximal, conséquence d'un important outrepassement: hormis un réfléchissement, les négatifs de lames sont parfaitement parallèles. Le débitage est unipolaire, mais la morphologie globale du nucléus n'est pas restituable. Le plan de frappe est droit, aménagé par de nombreux enlèvements centripètes. Les corniches sont supprimées et adoucies par abrasion.

- un fragment distal, réutilisé en nucléus à éclat. Les enlèvements sont toujours unipolaires, tournants sur les trois quarts de la surface. Le nucléus devait présenter une silhouette ogivale. Les enlèvements sont tous de largeur inférieure à dix millimètres, témoignant d'une exploitation plus poussée que les deux autres nucléus, avec la production de supports lamellaires. Ces supports de dimensions modestes ont d'ailleurs été identifiés parmi les produits de débitage.

La chaîne opératoire laminaire s'est déroulée sur le site, comme en témoignent les nombreux éclats de mise en forme associés à cette production. Des éclats corticaux sont également présents, mais il est difficile de savoir si les blocs ont été apportés bruts ou partiellement épannelés. indirecte s'avère toutefois largement majoritaire. Elle est utilisée lors de la mise en 
place des volumes du nucléus, notamment pour la préparation des crêtes. Elle est également majoritairement mise en œuvre pour le débitage, favorisant l'obtention de produits réguliers.

La mise en forme du bloc consiste en la mise en place de convexités préalablement à l'aménagement d'une crête et ce, dès la phase d'entame et d'initialisation. Cette première phase du débitage est représentée par 186 objets identifiés comme éclats d'initialisation associés à la préparation des nucléus à lames. Il s'agit d'éclats souvent corticaux à plus de $50 \%$, voire totalement; généralement fins et arqués, ces éclats ont majoritairement été obtenus en percussion indirecte. L'association de ces éclats à la première étape de la chaîne opératoire laminaire ne fait guère de doute. Des éclats corticaux témoignant d'une mise en place de la crête dès l'épannelage ont également été identifiés sur le site de Poses (Bostyn et al., 2003). Il s'agit véritablement d'une « initialisation pré-déterminante» qui rentabilise les convexités naturelles des rognons, optimisant ainsi le volume du bloc. Cette gestion se poursuit dans les phases initiales du débitage, illustrée par une lame à crête partielle et corticale (fig. 15, no 6). Lors du plein débitage, les convexités naturelles sont encore mises à profit pour élargir la table laminaire, comme l'attestent des lames présentant des pans corticaux (fig. 15, no 5) ainsi qu'un remontage entre deux produits laminaires débités dans le silex jaune orangé.

Les produits laminaires sont représentés par 110 produits ou fragments de produits laminaires, ainsi que 54 outils sur lame ; s'y ajoutent 9 fragments de lames à crête, ainsi qu'une lame sous crête. Les produits appartiennent majoritairement à la catégorie des lames, mais des lamelles (largeur $<10 \mathrm{~mm}$ ) ont aussi été débitées plus occasionnellement.

Les talons sont fréquemment lisses et punctiformes, les corniches étant toujours soigneusement réduites et abrasées. La gestion unipolaire par percussion indirecte est très largement prépondérante. Les lames obtenues sont régulières, avec trois ou quatre pans, pour une largeur moyenne oscillant entre 15 et $20 \mathrm{~mm}$. On remarque également quelques produits nettement plus larges $(30 \mathrm{~mm})$, plutôt rapportables à des phases d'entretien des tables (fig. 15, no 11). Enfin, des lames ou lamelles de largeur réduite ont également été produites, certainement en fin d'exploitation des nucléus.

\section{La production d'éclats}

71 La production d'éclats véritables est minoritaire au sein de l'assemblage $(12,5 \%)$. Les éclats sont débités dans le même silex que le reste de la série. La percussion dure est systématique. Le débitage est souvent multidirectionnel. Les nucléus ont souvent été poussés à un stade avancé d'exhaustion. On peut identifier quelques nucléus à éclat, exploitant d'anciens nucléus à lame, ces derniers étant d'ailleurs quasiment absents (tabl. 4). Ainsi, la production d'éclats, secondaire en nombre, semble avoir été réalisée, au moins pour une partie, consécutivement au débitage laminaire. Certains éclats massifs, épais et conservant de grandes plages de table laminaire, s'apparentent à des témoins de «destruction de nucléus à lame ». On peut se demander si les nucléus à lame n'ont pas été immédiatement débités en éclats, pour des raisons qui dépassent le simple besoin de supports ? Cette « mutilation » ou du moins ce re-débitage des nucléus à lame a aussi été observé à Poses (Bostyn et al., 2003). 
72 Une fois les convexités devenues impropres au débitage d'éclats, les nucléus ont souvent été transformés en percuteurs. L'amplitude des surfaces bouchardées est variable, parfois étendue ou parfois seulement cantonnée à quelques arêtes. Des éclats portant des surfaces bouchardées résiduelles attestent de la fracturation de ces pièces lors d'une percussion directe. La reconversion des nucléus en percuteurs peut palier une déficience en galets dans l'environnement du site. Aussi, une utilisation pour le débitage du silex est envisageable. Cette hypothèse est renforcée par l'absence de galets percutés dans l'assemblage.

\section{L'outillage}

73 On dénombre 103 outils en silex au sein de la série. L'outillage est réalisé dans des proportions comparables (tab. 3 et 4 ) sur lame (52\% de l'outillage) et sur éclat (44\%). L'outillage sur débris est marginal (4\%).

Les outils retouchés et un échantillon de produits «bruts » ont été l'objet d'une analyse tracéologique sous la conduite de Jean-Paul Caspar. Les données issues de ce travail ont été intégrées à ce texte, dans l'objectif de ne pas déconnecter les lectures typologique et fonctionnelle.

Tableau 3 : Les différentes catégories avec la part de l'outillage.

Table 3: Different categories of lithic artefacts with the tools group.

Tableau 4 : Décompte du mobilier lithique par structures et pourcentages par catégories. Table 4: The lithic artefacts by feature and the percentages by category.

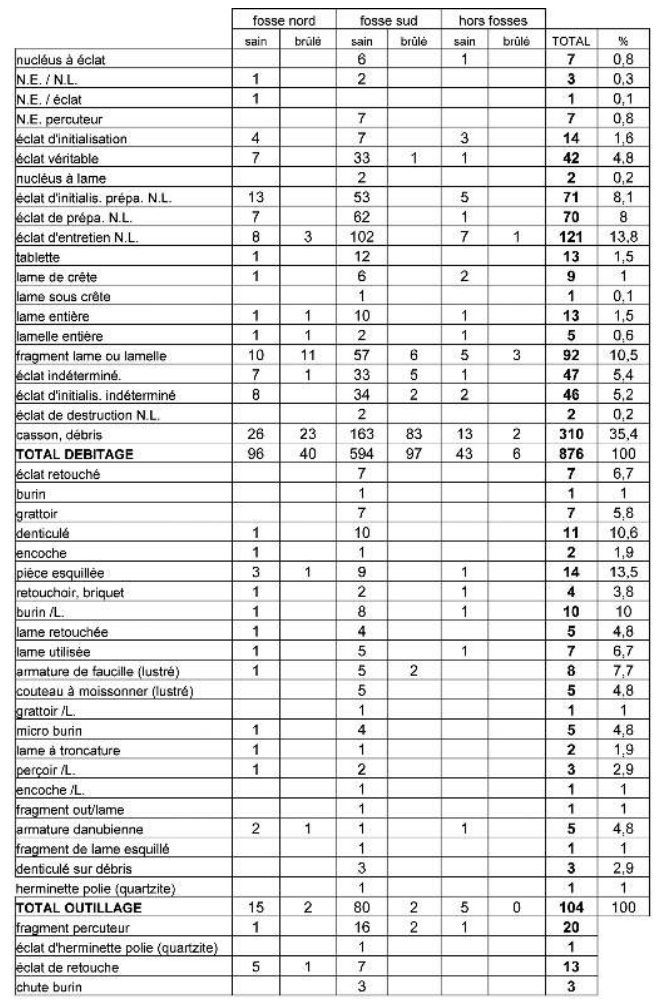




\section{L'outillage sur lame}

Les pièces à lustré réunissent deux types d'objets : huit armatures de faucille (fig. 13, no 1 à 8) dont le lustré, souvent prononcé, envahit plus ou moins les deux côtés d'une extrémité de la pièce, trahissant l'emprise de l'emmanchement, et cinq fragments de lame (fig. 13, no 9 à 12) qui portent un lustré plus marginal, affectant une mince bande sur le long du bord de la face généralement inférieure de la pièce.

Figure 13 : Armatures de faucille et autres lames à lustré. Dessins L. Juhel.

Figure 13: Sickle blade inserts and other polished blades. Drawings L. Juhel.
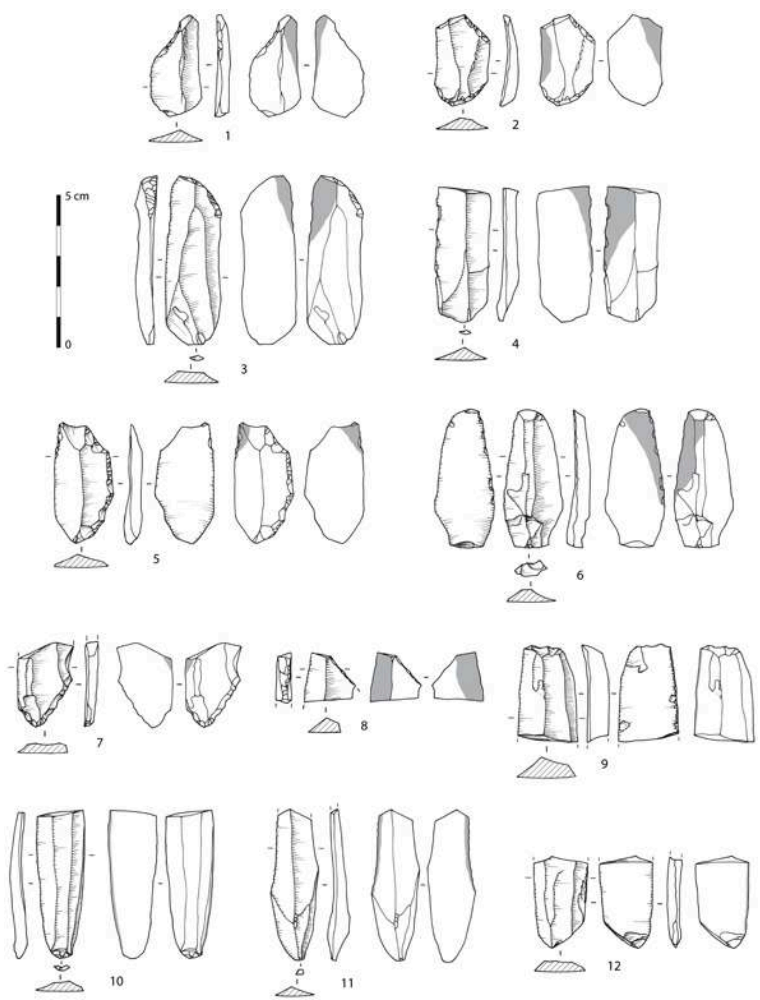

La typologie des armatures de faucilles est variée. Elles sont réalisées sur des fragments de lames, exploitant des cassures peut être liées au débitage (fig. 13, no 4), ou aménagées par troncature simple ou double, et généralement oblique du côté emmanché. La retouche est toujours directe, parfois abrupte. Trois pièces sont obtenues par la technique du micro-burin (fig. 13, no 1, 2 et 5), deux d'entre elles utilisant les segments à piquant trièdre; la fracturation de la troisième est atypique (pas de piquant trièdre). autres.

On peut entrevoir deux modules au sein de ces armatures: un module assez court $(\mathrm{L}<35 \mathrm{~mm})$ pour trois d'entre elles, tandis que quatre autres sont relativement allongées (jusqu'à $55 \mathrm{~mm}$ ); dans ce dernier cas, elles conservent toutes le talon du support laminaire initial.

Les lames à lustré marginal sont réalisées à partir de supports très réguliers issus du plein débitage. Elles présentent peu d'aménagements significatifs, d'autant plus que 
trois d'entre elles sont fragmentaires, du fait de cassures anciennes ( 3 cas) ou récente ( 1 cas) ; la cinquième a subi une forte détérioration par une exposition au feu.

Deux lames conservent leurs talons (fig. 13, no 10 et 11), tandis qu'une autre comporte une retouche inverse envahissante de son extrémité distale (fig. 13, no 12). Une pièce est affectée d'une troncature accompagnée d'un esquillement de la face inférieure.

L'examen tracéologique des différents types de pièces à lustré confirme pour l'ensemble une action de découpe de plantes non ligneuses à l'état frais. Dans le cas des armatures de faucille, la plage lustrée macroscopique, disposée en oblique par rapport à l'axe morphologie, témoigne d'une disposition classique "en épi » des éléments lithiques dans les manches.

82 On soulignera également l'absence de recyclage de ces lames à lustré dans d'autres chaînes d'activités.

83 Les burins (10 pièces) ont été réalisés préférentiellement sur fragment mésial ou proximal des lames. Ces lames fragmentaires, assez nombreuses dans la série, peuvent être une conséquence accidentelle de la technique de débitage indirect. Tous sont des burins d'angle. On dénombre quatre burins multiples et six pièces ont été l'objet de raffûtages.

84 Sept pièces ont été fabriquées sur des lames de plein débitage, majoritairement sur cassure ( 7 pièces) ou parfois sur troncature ( 3 pièces). Des lames moins régulières et conservant des lambeaux de surface corticale ont également été utilisées : deux pièces sont réalisées sur troncature (fig.14, no 5 et 14). Un burin multiple (4 enlèvements) est réalisé sur troncature pour la partie proximale du support et sur cassure pour l'extrémité distale (fig. 14, no 13). 
Figure $14: 1$ à $4:$ mèches et perçoirs ; 5 : fragments de burin ; $6:$ pièce à dos abattu ; 7 à 14 : burins. Dessins L. Juhel.

Figure 14: 1 - 4: piercers; 5: fragments of burins; 6: edge pieces; 7 - 14: burins. Drawings L. Juhel.
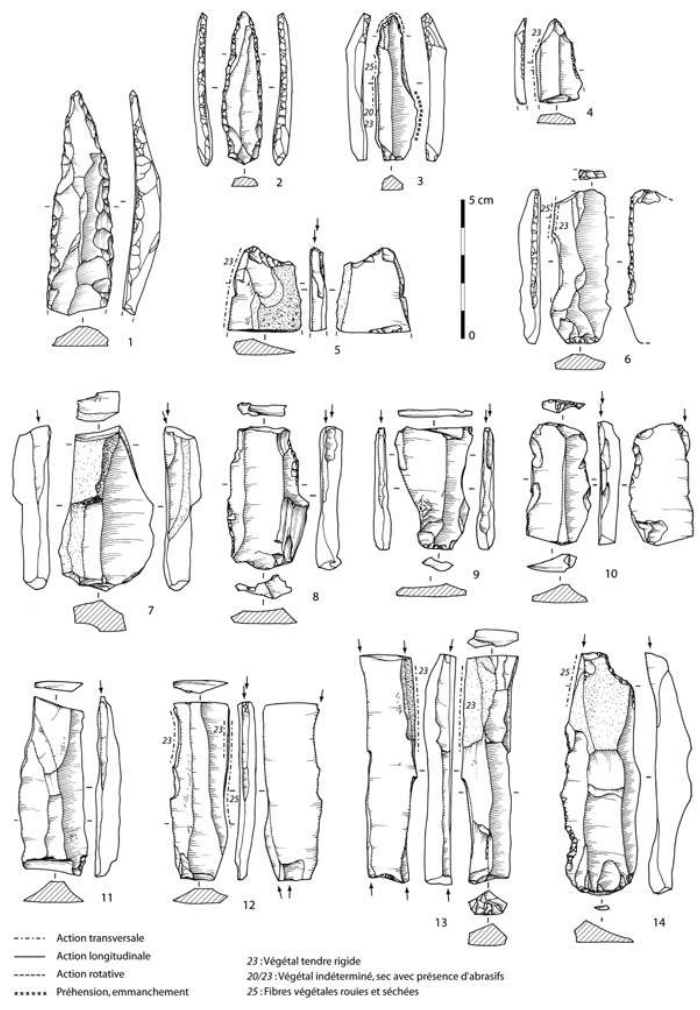

Sur le plan fonctionnel, cinq pans de burin témoignent d'un travail des végétaux tendres rigides, tels que les phragmites ou les roseaux (poli 23) (le code « 23 » est ici réservé au lustre marginal brillant ondulé. Le code « 25 » désigne les stigmates rapportés au teillage de fibres rouies et séchées). Ces traces sont parfois associées à des stigmates interprétés comme le travail de fibres rouies et séchées (poli 25), rapportables à une activité de teillage (Caspar et al., 2003).

Les lames "utilisées» (7 pièces) forment une catégorie qui a été identifiée par une lecture macroscopique. Il s'agit majoritairement de lames régulières de plein débitage et de quelques pièces techniques (lames à crête), non retouchées, mais présentant de fins esquillements des bords (fig. 15, no 1 à 11).

L'ampleur de cette catégorie doit toutefois être majorée au vu des résultats de l'analyse microscopique. Les lames utilisées brutes témoignent du travail des matières animales et végétales. Trois lames présentent les marques d'une découpe brève dans des tissus carnés tendres (peau, viande, ligaments...) sur un ou deux bords (fig. 15, no 9). Une autre lame porte des traces similaires à celles obtenues par un dépeçage expérimental. Le travail des végétaux inclut la découpe de végétaux non ligneux (catégorie rapportable aux armatures de faucille) mais aussi le travail de végétaux indéterminés à l'état sec et en présence d'abrasif (poli 20/23) et, également, des usures rapportées au teillage. 
Figure $15: 1$ à 11 : débitage laminaire ; 12 : nucléus à lames. Dessins L. Juhel. Figure 15: 1 - 11: blade debitage ; 12: blade core. Drawings $L$. Juhel.

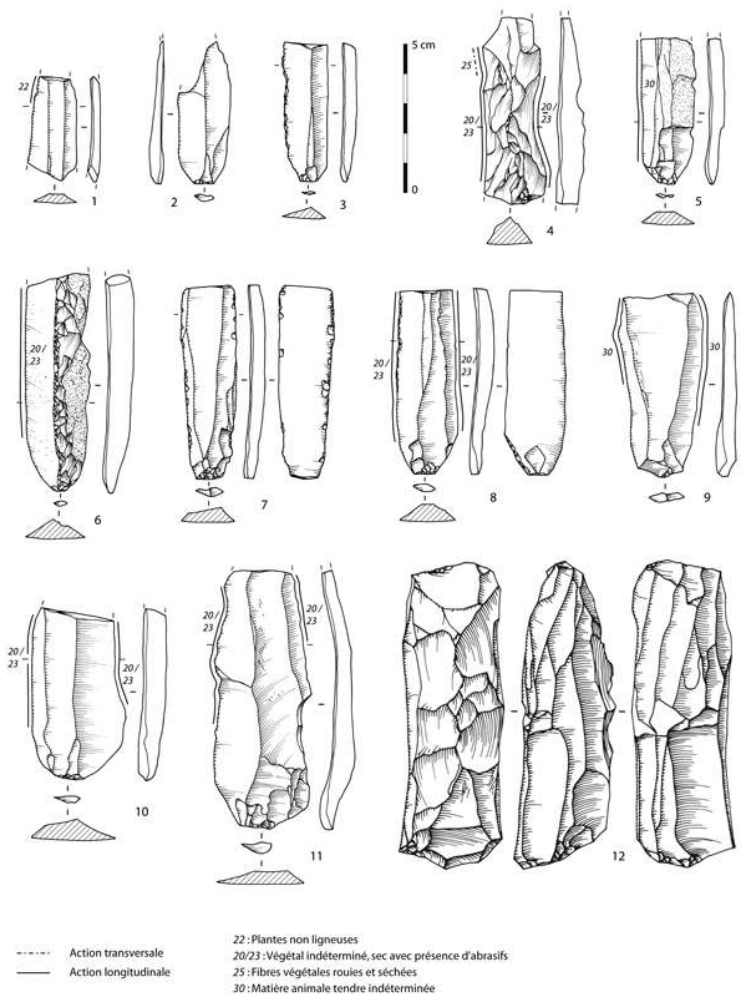

Les lames retouchées (5 pièces) sont des lames affectées le plus souvent de retouches marginales ou atypiques. Le bord d'une lame a été abattu par une retouche inverse (fig. 14, no 6). Une autre lame voit son extrémité proximale affectée par une petite retouche inverse, accompagnée d'un fort émoussé sur l'ensemble du talon (fig. 15, no 8).

Les micro-burins ( 5 pièces) sont issus de la segmentation de lames ou de lamelles pour la fabrication des armatures de flèche ou de faucille utilisant des piquants trièdres. Trois pièces sont des micro-burins proximaux, réalisés sur des lames régulières d'épaisseur variable (fig. 16, no 6 à 8).

Les armatures perçantes (5 pièces) sont toutes asymétriques et triangulaires. Elles sont fabriquées à partir de la technique du micro-burin et présentent les caractéristiques suivantes :

- latéralisée à droite, avec une partie distale façonnée par un piquant trièdre et une troncature oblique légèrement irrégulière et une troncature directe et oblique à l'axe du débitage, en retouche abrupte rectiligne (fig. 16, no 1);

- latéralisée à droite, avec une partie distale façonnée par un piquant trièdre et une troncature oblique rejointe par la troncature arrondie de la base, en retouche directe abrupte et inverse envahissante (fig. 16, no 5);

- latéralisée à gauche, avec une partie distale façonnée par un piquant trièdre et une troncature oblique rectiligne et en partie proximale une troncature légèrement convexe, en retouche directe abrupte et inverse envahissante (fig. 16, no 2 et 4);

- latéralisée à gauche, avec une troncature oblique obtenue par une cassure atypique de micro-burin et une base à troncature oblique, en retouche directe (fig. 16, no 3). 
L'examen tracéologique confirme qu'aucune armature ne présente d'abrasion de la base. Les traces d'impact sont également absentes.

Figure $16: 1$ à 5 : armatures perçantes ; 6 à $8:$ micro-burins. Dessins L. Juhel. Figure 16: 1 - 5: sharp blade inserts; 6 - 8: micro burins. Drawings L. Juhel.
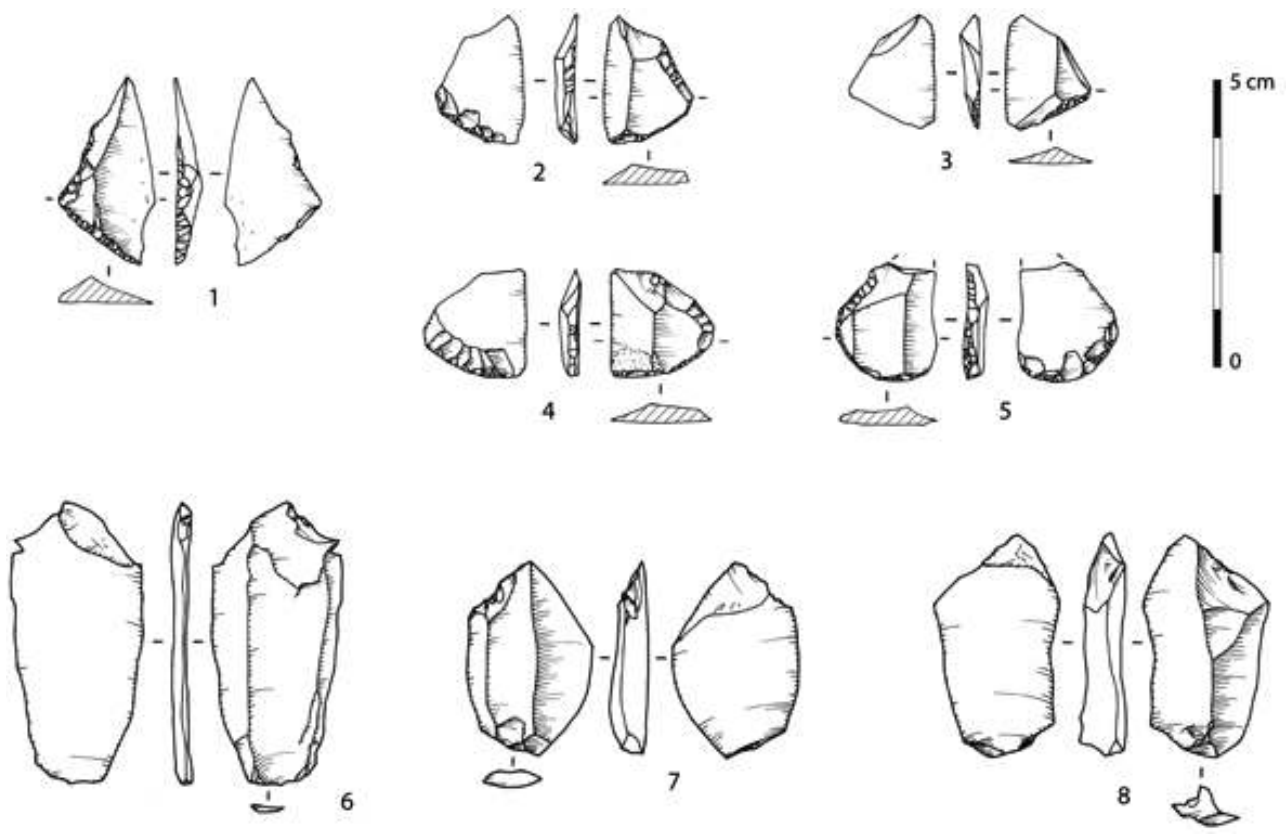

Les perçoirs (3 pièces) : deux sont réalisés sur des lames de plein débitage. La retouche abrupte ou semi-abrupte est directe et convergente, sur la longueur totale des deux bords. La grande régularité et le profil relativement droit d'un exemplaire permettent son interprétation en mèche de foret (fig. 14, no 2). Pour le deuxième, le profil assez cintré rend moins probable ce type d'utilisation emmanchée (fig. 14, no 1). Le troisième est réalisé sur une lamelle assez épaisse, et peu arquée (fig. 14, no 3). Seule l'extrémité a subi une retouche abrupte convergente dégageant la pointe. De petits esquillements inverses trahissent une utilisation par mouvement rotatif ; là encore, l'emmanchement en mèche de forêt est possible, mais la tracéologie révèle des fonctions multiples et peut-être déconnectées pour cet objet. Un bord porte des traces de préhension ou d'emmanchement, tandis que le côté opposé témoigne du travail de matériaux végétaux.

Les troncatures ( 2 pièces) sont des fragments de lames tronquées par une retouche abrupte faiblement oblique.

Le grattoir (1 pièce), en silex bathonien jaune, est réalisé sur un éclat laminaire partiellement cortical, originaire de la phase d'initialisation du nucléus (fig. 17, no 2). Une petite série de retouches aménage un front abrupt, faiblement convexe, dont la partie gauche est encore corticale. La base de la pièce a subi une retouche directe sur un côté, et inverse sur le bord opposé. Cet aménagement peut avoir été effectué pour faciliter un emmanchement. 
Figure $17: 1$ à $6:$ grattoirs ; 7 à $11:$ denticulés. Dessins L. Juhel. Figure 17: 1 - 6: scrapers; $7-11$ : denticulates. Drawings L. Juhel.
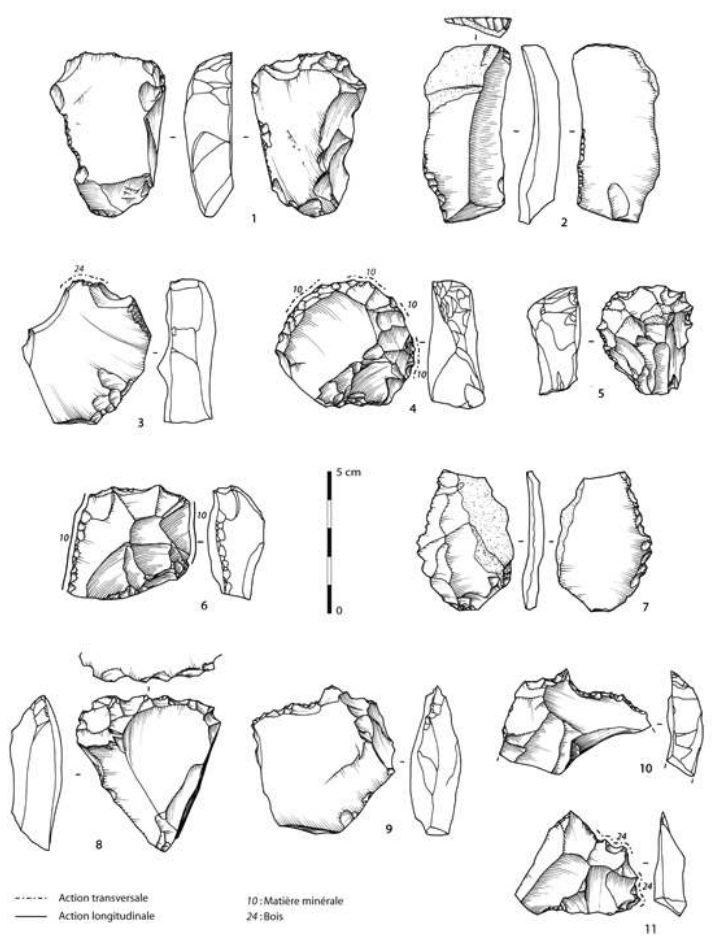

La lame à encoche (1 pièce) est un fragment proximal de lame, dont une retouche inverse détermine une encoche profonde sur la face inférieure.

La pièce esquillée (1 pièce) est un fragment de lame à crête en opalite, (fig. 18, no 4). Ses extrémités distale et proximale comportent des esquillements ainsi que des enlèvements burinants, d'origine accidentelle, sans doute liée à une utilisation en percussion indirecte (type coin). Cette utilisation est certainement secondaire pour ce produit laminaire d'importation qui a dû avoir un usage initial différent.

\section{L'outillage sur éclat}

Les pièces esquillées (14 pièces) sont des éclats épais, de 15 à $27 \mathrm{~mm}$, à tendance allongée dans certains cas. Quelques supports appartiennent à la chaîne opératoire laminaire, mais la majorité est issue de la production d'éclats. Les tranchants esquillés présentent des angulations variant entre 25 et 45 degrés et exceptionnellement supérieures à 50 degrés. Les esquillements d'utilisation s'apparentent à des retouches écailleuses, unifaciales ou bifaciales. Elles se localisent le plus souvent en position distale et proximale (fig. 18, no 5 et 6) ou parfois latérale ou périphérique (fig. 18, no 7). Dans les cas des pièces à retouches opposées, une utilisation en percussion posée indirecte, de type coin à fendre, est vraisemblable.

Les denticulés (11 pièces) sont réalisés sur des éclats peu réguliers, dont l'épaisseur varie de $5 \mathrm{~mm}$ jusqu'à $28 \mathrm{~mm}$. Néanmoins, une moyenne se situe entre 15 et $20 \mathrm{~mm}$.

Les morphologies sont très variées (fig. 18, no 7 à 11), probablement à l'instar de leurs usages. Notons une pièce à retouches directes et inverses (fig. 18, no 8). Un autre exemplaire (fig. 18, no 6) est réalisé sur un support fin, partiellement cortical (éclat 
d'aménagement de crête). Une série de fines retouches inverses y façonnent un bord très régulièrement denticulé s'apparentant à une scie.

Figure $18: 1$ à $3:$ herminette ; $4:$ pièce esquillée en opalite ; 5 à $7:$ denticulés. Dessins L. Juhel. Figure 18: 1 - 3: adze; opalite splintered element; 5 - 7: denticulates. Drawings $L$. Juhel.
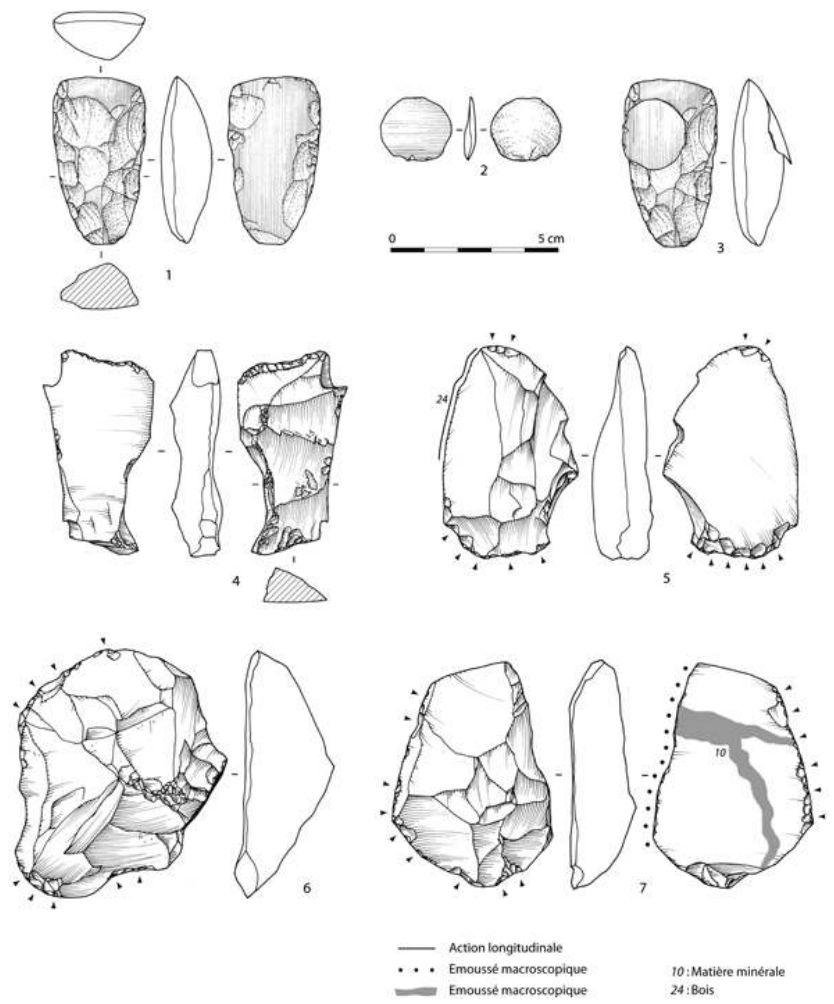

Les éclats retouchés (7 pièces) sont des éclats de morphologies variées, affectés de retouches atypiques, pouvant être accidentelles ou liées à de l'utilisation.

Les grattoirs (6 pièces) sont réalisés sur des éclats courts d'épaisseur moyenne (entre 15 et $20 \mathrm{~mm})$; un support est plus épais ( $27 \mathrm{~mm})$.

Une pièce s'apparente au grattoir herminette (fig. 17, $n^{\circ} 1$ ) tel qu'il a été identifié au sein du groupe de Bliquy (Caspar et Burnez-Lanotte, 2003). Le front est affecté par des esquillements et quelques retouches inverses, liées à une utilisation en percussion lancée. Un bord a été façonné par quelques enlèvements, allongeant ainsi la silhouette de l'objet. L'extrémité opposée au front actif a elle aussi subi des esquillements, ainsi que les deux arêtes latérales; ces stigmates évoquent un emmanchement. Une pièce au front peu large est dégagée par deux encoches travaillées du bois (fig. 17, $\mathrm{n}^{\circ} 3$ ).

Trois grattoirs illustrent quant à eux le travail du schiste. L'un a scié ce matériau à l'aide des deux côtés latéraux esquillés (fig. 17, $\mathrm{n}^{\circ} 6$ ), les deux autres l'ont gratté, probablement en va-et-vient au vu des nombreux micro-arrachements inverses des fronts actifs. Ces pièces sont très similaires aux outils et grattoirs sur éclat de Vaux-etBorset (Caspar et Burnez-Lanotte, 2003; Burnez-Lanotte et Caspar, 2005). Les ressemblances concernent également la morphologie et le degré de finition des fronts. Ils sont étroits, à la limite de l'ogival, ou circulaires (fig. 17, $n^{\circ} 4$ ), généralement étendus au trois quarts de la périphérie, voire plus. Dans ce dernier cas, il s'agit non pas d'un front continu, mais d'une succession de fronts courts adjacents, séparés par une petite 
encoche et utilisés indépendamment les uns des autres. Les « épines » permettent une meilleure pénétration dans la matière minérale.

Les «retouchoirs» (4 pièces) sont des éclats épais présentant une ou plusieurs extrémités ou arêtes latérales bouchardées, suite à une utilisation en percussion lancée sur un matériau dur. Ils ont pu être utilisés pour la retouche du silex et éventuellement, dans certaines phases de son débitage.

\section{Un outil poli}

L'herminette est un petit outil réalisé en quartzite blanc (fig. 18, $\mathrm{n}^{\circ}$ 1). Un petit éclat poli du même matériau a été découvert dans la même fosse (fig. $18, \mathrm{n}^{\circ} 2$ ). Le remontage de cet éclat sur la face supérieure de l'herminette (fig. 18, n 3 ) témoigne de la réfection des côtés de cet outil, par une retouche oblique sur la face "supérieure " (face convexe), accompagnée de quelques enlèvements inverses sur la face "plane». Un polissage très partiel a ensuite adouci les arêtes des négatifs d'enlèvements sur les deux faces. Cette réfection a en revanche épargné le tranchant qui porte par ailleurs plusieurs esquillements et une amorce d'enlèvement sur la face plane. Ces stigmates évoquent une utilisation emmanchée, en percussion lancée.

La réfection peut être intervenue consécutivement à une cassure mésiale ou proximale de l'outil, ou encore pour adapter celui-ci à un dispositif d'emmanchement (gaine ?). La réfection témoigne en tout cas d'une volonté de prolonger la durée de vie de cet outil, ce qui n'est guère surprenant pour une pièce polie en matériau exogène.

\section{Bilan de l'outillage}

107 La série présente un relatif équilibre entre outils sur éclat et outils sur lame, ces derniers étant très légèrement supérieurs en nombre. Le caractère anecdotique de l'utilisation des débris s'explique par le faible nombre de supports potentiels et de dimensions suffisantes au sein de cette catégorie.

Une variation qualitative des supports laminaires est perceptible au sein des différentes catégories d'outils. Les lames de plein débitage les plus régulières sont utilisées préférentiellement pour la fabrication des armatures de faucille et de couteau à moissonner, des armatures de flèche et des perçoirs de type mèche. Les lames « utilisées » font aussi partie des supports les plus réguliers. Par contre, les burins sont réalisés sur des lames moins régulières, parfois corticales, souvent rapportables à des étapes de mise en forme ou d'entretien du nucléus. Pour certaines d'entre elles, le choix du support a sans doute été motivé par l'épaisseur, déterminante pour la morphologie des pans et des dièdres, plutôt que par la régularité.

L'outillage sur éclat exploite, quant à lui, préférentiellement les éclats "véritables ", mais des éclats d'initialisation ou de mise en forme laminaire sont souvent utilisés, ainsi que quelques débris. Le choix d'un support apparaît donc relativement opportuniste, mais l'objectif fonctionnel s'avère toujours en adéquation avec la morphologie du support.

Sur le plan fonctionnel, on remarque un taux d'utilisation particulièrement élevé pour la catégorie des lames brutes (25 utilisées sur un échantillon de 35 pièces). Dans le cas des éclats bruts, cinq pièces ont révélé des traces d'utilisation, sur une sélection de vingt-trois supports. L'étude tracéologique révèle des activités variées, parmi lesquelles 
le travail des végétaux non ligneux tient une place importante, incluant le travail des fibres végétales rouies et séchées. Le travail du bois et la découpe de matières animales sont plus discrètement représentés. Enfin, le travail du schiste par des outils de type grattoir est peut-être mis en relation avec les ébauches de bracelet, confirmant une fabrication de ces derniers sur le site.

\section{Conclusion de l'étude de l'industrie lithique}

La série lithique de Fontenay-le-Marmion appartient résolument à une occupation domestique du Néolithique ancien. Le corpus est dominé par trois catégories : les pièces esquillées, les denticulés et les burins sur lame. Les considérations statistiques doivent être abordées avec précaution car le nombre d'outils reste modeste et peut refléter des réalités fonctionnelles à l'échelle du site plutôt que de véritables tendances culturelles. Quelques remarques peuvent néanmoins être proposées. Les burins sur lame $(10 \%)$ et les pièces esquillées $(13,5 \%)$ dominant largement les grattoirs sur éclat $(5,8 \%)$ rappellent les assemblages anciens ou rapportés au Rubané récent du Bassin parisien. En effet, les pièces esquillées s'infléchissent au Villeneuve-Saint-Germain, au profit des grattoirs sur éclat qui deviennent majoritaires dans les assemblages du Néolithique moyen (Bostyn, 1994 ; Augereau, 2004). Mais ces rapprochements avec l'univers rubané restent limités, reflétant seulement la perduration de traditions techniques et typologiques. On note d'ailleurs l'absence de véritable grattoir sur lame, pourtant présent dans les séries anciennes. Inversement, aucun outil n'est caractéristique des séries tardives. L'absence de tranchet ou d'armature tranchante empêche le rapprochement avec les assemblages du type VSG « à cordon et tranchet ». Finalement, sur le plan culturel, l'outillage s'apparente à un faciès classique, tout à fait rapportable au VSG moyen.

La série trouve un écho local au travers de l'assemblage de la maison de Mondeville (Chancerel et al., 2006) qui, elle aussi, a livré une centaine d'outils. L'outillage y est très comparable avec une prépondérance des armatures de faucille et des burins. Des grattoirs sur éclat irréguliers et parfois denticulés sont présents, rappelant la typologie des exemplaires de Fontenay-le-Marmion. Les denticulés y sont par contre minoritaires, et les auteurs ne répertorient pas de pièces esquillées. Enfin, la présence d'un tranchet (Chancerel et al., 2006) y apparaît anecdotique, au même titre que l'herminette polie présente à Fontenay-le-Marmion.

113 Les cinq armatures de flèche constituent un petit corpus qui représente un jalon important au niveau régional. Ces armatures perçantes à piquant trièdre se rattachent parfaitement à la tradition danubienne. Mais on peut noter l'apparition d'un type bien spécifique, dont la retouche inverse et rasante détermine une base arrondie pour au moins deux exemplaires. Ces armatures à base arrondie sont bien représentées mais mal datées dans l'abri sous roche d'Omonville-la-Petite (Juhel et al., 2006) où elles sont également réalisées en silex jurassique de la plaine de Caen, ici importé en contexte armoricain. Le tumulus des Fouaillages à Guernesey en a également livré un exemplaire comparable (Marcigny et al., 2010). Le site de Fontenay-le-Marmion peut aujourd'hui faire office de référence chronologique pour ces pointes dont la typologie particulière pourrait avoir une signification géographique et culturelle dans le Nord-Ouest de la France. 
114 Sur le plan du débitage, l'assemblage de Fontenay-le-Marmion se caractérise par l'importance de la chaîne opératoire laminaire. Mais, comme pour l'outillage, il semble opportun de relativiser la représentativité statistique de l'échantillon. Le mobilier découvert peut en effet provenir de rejets ponctuels, liés à une production spécifique mais finalement non représentative de l'activité du site ou de l'unité d'habitation dans la durée. Il reste difficile d'appréhender ces notions à partir des faits archéologiques. Néanmoins, il est avéré que des lames de qualité et impliquant un savoir-faire associé à un matériau particulier ont été produites sur le site. Les nombreuses lames utilisées puis rejetées avec les déchets de leur production illustrent clairement une production destinée à subvenir aux besoins de l'habitat. Il est impossible, au travers de cet assemblage, d'identifier une éventuelle production excédentaire aux besoins domestiques, qui aurait pu être l'objet d'une diffusion, telle qu'elle est connue vers les sites armoricains par exemple. Peut-on penser que cette production serait plutôt à rechercher directement sur les gisements de matière première ? Les ateliers éponymes de la commune des Moutiers-en-Cinglais, reconnus dans les années quatre-vingts, sont distants d'une dizaine de kilomètres de l'habitat de Fontenay-le-Marmion. Dans ce secteur, l'importance des artefacts liés à la production laminaire témoigne vraisemblablement d'une production spécialisée hors habitat, et associée aux gites de matière première. À quelques kilomètres de là, l'habitat de Fontenay-le-Marmion a été la résidence de tailleurs détenteurs d'un savoir-faire laminaire bien perceptible dans la série domestique. Il est, de ce fait, tentant d'imaginer, à titre d'hypothèse, que ces individus ont pu ponctuellement exercer sur des «ateliers " associés aux sources de silex proches du site, produisant des lames destinées à alimenter des réseaux d'échange et de circulation...

\section{La parure}

\section{Les pièces en schiste}

\section{Aspects quantitatifs et qualitatifs}

Quatorze fragments d'anneaux en schiste ont été découverts sur le site: sept proviennent de la fosse sud F6, trois de la fosse sud F25, deux sont issus de la fosse nord (F7). Les deux derniers ont été trouvés au sud de l'unité d'habitation, près du « chemin Haussé ». À l'exception des pièces hors contexte et celle découverte lors du décapage en surface de F25, leur état de conservation est correct.

La simple observation macroscopique permet de distinguer au moins cinq matériaux différents, avec toute la prudence que cela impose (fig. 19). Le schiste tacheté est le plus utilisé, avec six anneaux. Trois pièces sont confectionnées dans un schiste mat foncé (couleur ardoise), peut-être du schiste du Pissot. Le troisième matériau, utilisé pour deux anneaux, correspond à du grès schisteux briovérien. Un fragment d'anneau est fabriqué dans un schiste noir à inclusion de feldspath et un autre dans un schiste à litage brillant (effet vermiculé). 

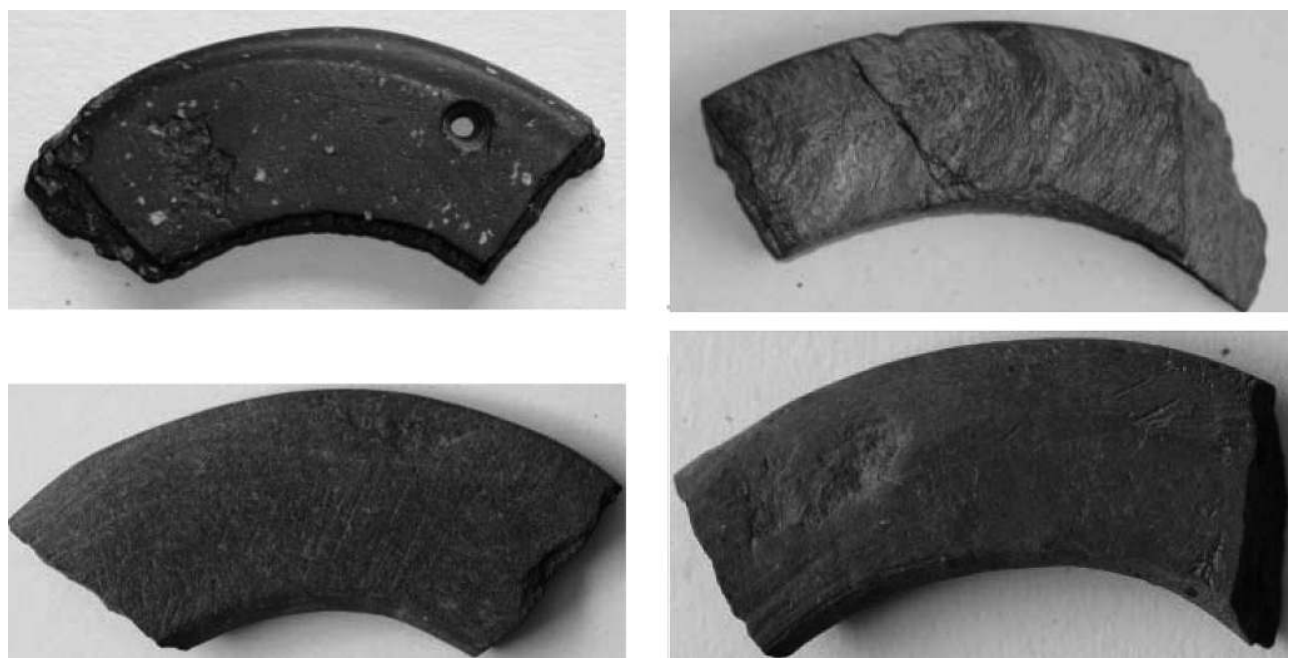

Figure 19 : Exemples de fragments d'anneaux en différents schistes. Clichés P. Giraud. Figure 19: Fragments of rings of different types of schist. Photos P. Giraud.

117 On constate donc une provenance variée de la matière première. Toutefois, ces schistes se retrouvent dans différents gisements du Massif armoricain (Fromont, 2003). Les schistes briovériens, dont des affleurements sont localisés à moins de deux kilomètres du site, n'arrivent qu'en troisième position alors que les gisements de schistes tachetés, les plus nombreux, sont éloignés de plusieurs dizaines de kilomètres, au nord du département de l'Orne (Athis) ou dans la région de Vire dans le Calvados.

\section{Les pièces de la chaîne opératoire (fig. 20)}

118 Quatre éléments font partie de la chaîne opératoire de la fabrication de bracelets, il s'agit de trois disques réguliers et d'une ébauche (bracelet $\mathrm{n}^{\circ} 1$ ). 
Figure 20 : Pièces de la chaîne opératoire. Dessins A. Cocollos et L. Juhel. Figure 20 : Elements of the production chain. Drawings A. Cocollos and L. Juhel.

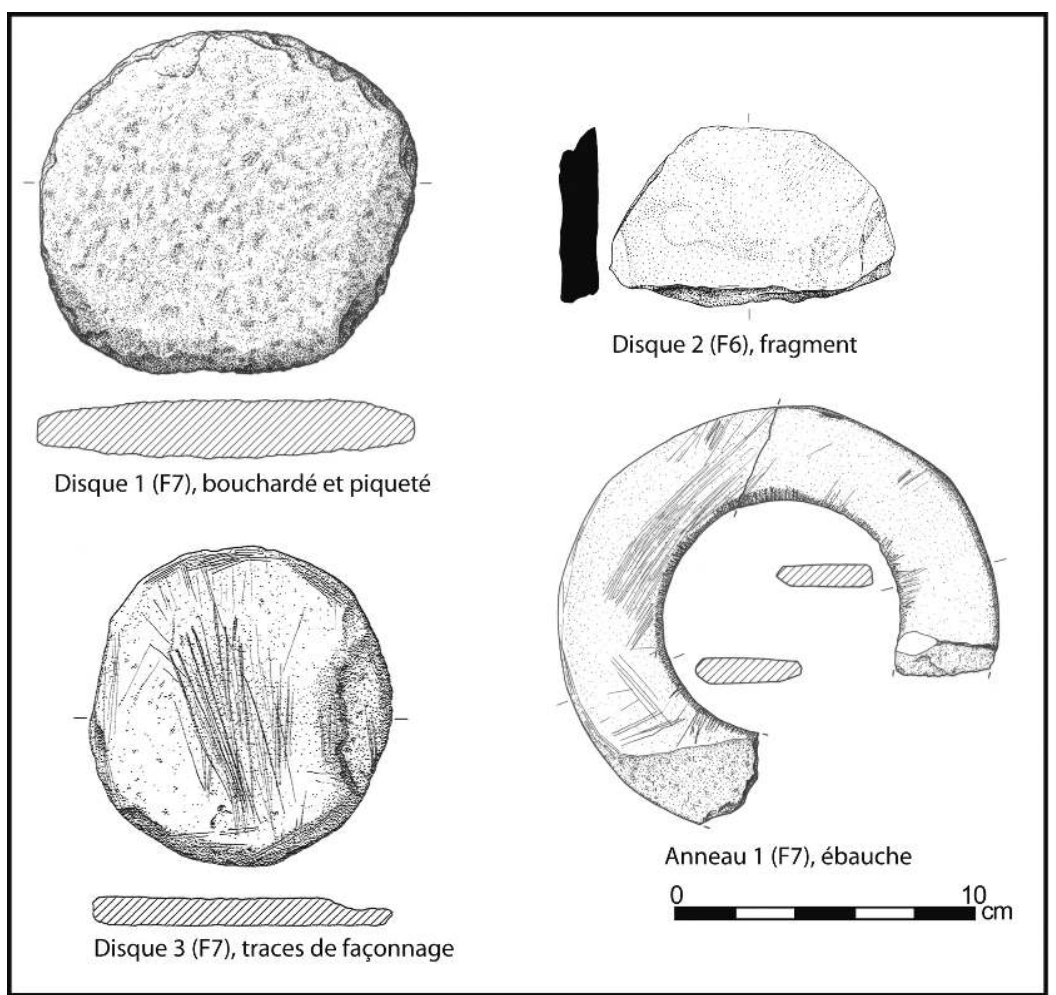

119 Le disque 1 a été mis au jour lors du diagnostic archéologique et provient de la fosse nord. Il est de forme ovalaire ( $\mathrm{L}: 12,6 ; 1: 11,5)$ et son épaisseur maximale mesure deux centimètres. Il présente des traces de bouchardage sur les deux faces et sa périphérie. La matière première utilisée est un schiste tacheté.

Le disque 2 est une moitié de disque découvert dans la fosse sud F6. Son diamètre est estimé à 9,5 cm pour une épaisseur de $1,7 \mathrm{~cm}$. Les traces de taille ou de façonnage ne sont pas évidentes ; il semble qu'il ait été grossièrement taillé sur le pourtour. C'est un schiste tacheté qui a la particularité d'être d'aspect varié : il présente des auréoles de matière fine uniforme et d'autres à forte concentration de taches foncées.

Le disque 3 a un degré de finition beaucoup plus avancé que les deux précédentes .Il est issu de la fosse nord (diagnostic). D’abord considéré comme le résidu de la découpe d'un anneau, son diamètre supérieur à $10,5 \mathrm{~cm}$ le classe plutôt parmi les disques. Des traces de façonnage et de régularisation sont visibles. Sur le pourtour, nous pouvons observer quelques stries verticales et horizontales et des rayures parfois profondes sont présentes sur les deux faces. Il s'agit probablement d'un schiste tacheté.

Le bracelet 1 est-il une ébauche? Cet anneau également découvert pendant le diagnostic, dans la fosse nord, semble être un bracelet en cours de fabrication. Sa forme est encore irrégulière, la largeur de sa couronne varie de 3,3 à 3,6 centimètres. L'arrondi n'est pas parfait et des traces de façonnage sont visibles sur le pourtour (stries obliques). La découpe de l'intérieur de l'anneau se lit aisément, par la présence de nombreuses traces saillantes segmentées. La surface de l'anneau est irrégulière et comprend une multitude de fines stries de polissage grossier. La matière première utilisée est également un schiste tacheté. 
La plupart des anneaux découverts sur ce site comporte des traces de fabrication (fig. 21). Les stries de découpe du disque interne sont visibles sur pratiquement toutes les pièces ; à l'exception de celles du bracelet 3 (verticales), toutes forment une section segmentée. Il est courant, sur les sites d'habitat, de trouver des bracelets dont le bord interne n'est pas ou peu poli. Il ne s'agit pas pour autant d'ébauches. Plusieurs d'entres eux comprennent d'ailleurs une perforation, peut-être réalisée pour effectuer une réparation du bracelet.

Figure 21 : Traces de fabrication. Clichés P. Giraud. Figure 21: Traces of production. Photos P. Giraud.

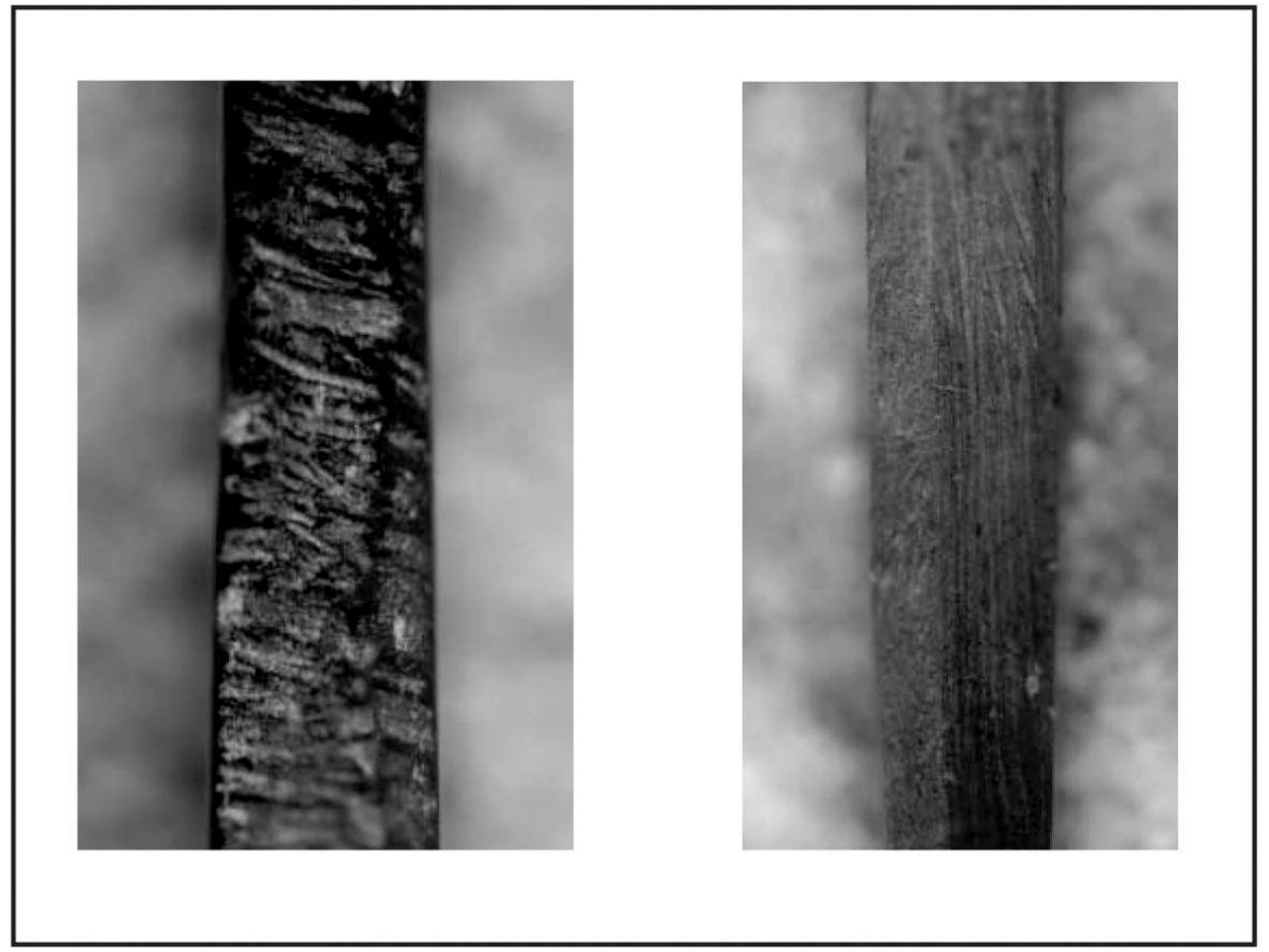

Les éléments évidents de la chaîne opératoire de la fabrication de bracelets - à savoir les disques réguliers et l'ébauche - sont tous en schiste tacheté, provenant probablement de gisements du nord du département de l'Orne. Malgré la proximité de gisement des grès schisteux briovériens, aucun indice de fabrication d'anneau dans ce matériau n'est attesté sur le site. Les nombreux éléments en grès briovérien mis au jour semblent, pour la plupart, destinés aux fonctions de polissage et à la mouture. La dizaine de petits éclats de schiste découverts dans les fosses latérales ne portent pas la trace évidente de stigmates de taille ou de polissage. Aucun d'entre eux n'est d'ailleurs en schiste tacheté.

\section{Les traces de réparation}

Les bracelets 3, 5, 9 et 14 sont perforés, La perforation de l'un d'eux $\left(n^{\circ} 9\right)$ pourrait être inachevée (fig. 22).

Typologie des bracelets (fig. 22) 
Figure 22 : Planche des fragments d'anneaux en schiste. Dessins A. Cocollos. Figure 22: Fragments of schist rings. Drawings A. Cocollos.

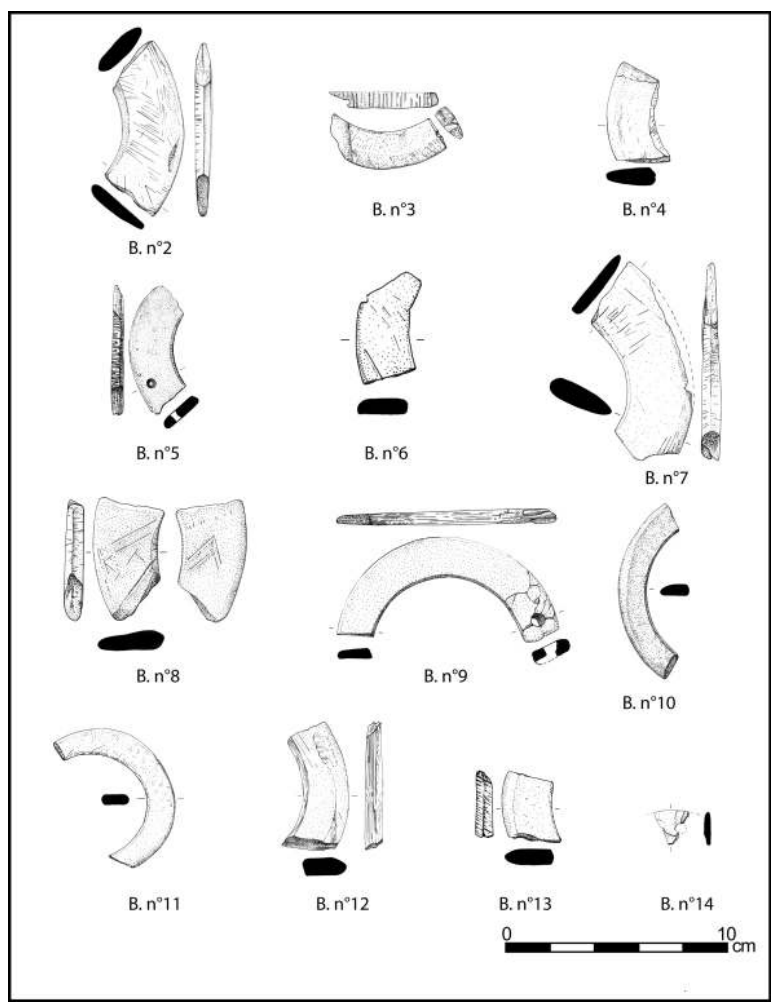

Les dimensions (tabl. 5 ; fig. 23)

126 À l'exception du bracelet $n^{\circ} 14$, trop fragmentaire, nous avons pu prendre les mesures des différentes pièces : diamètres, largeurs de couronne et épaisseurs.

Figure 23 : Graphiques comparatifs des dimensions des anneaux en schiste. Figure 23: Comparative graphs of the sizes of schist rings.

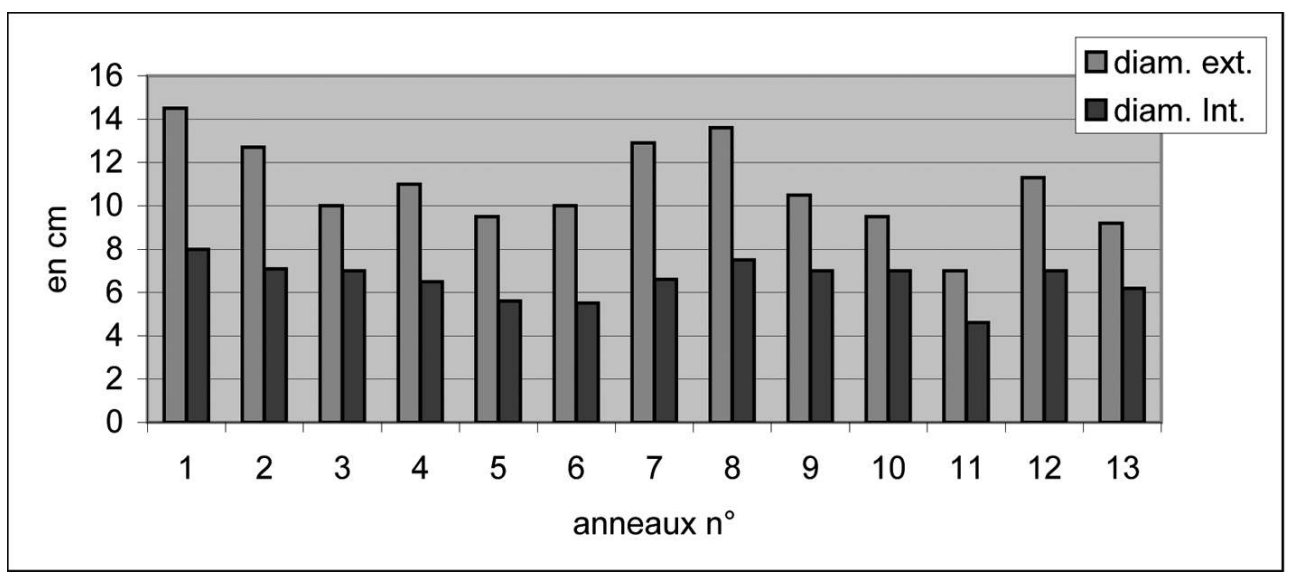




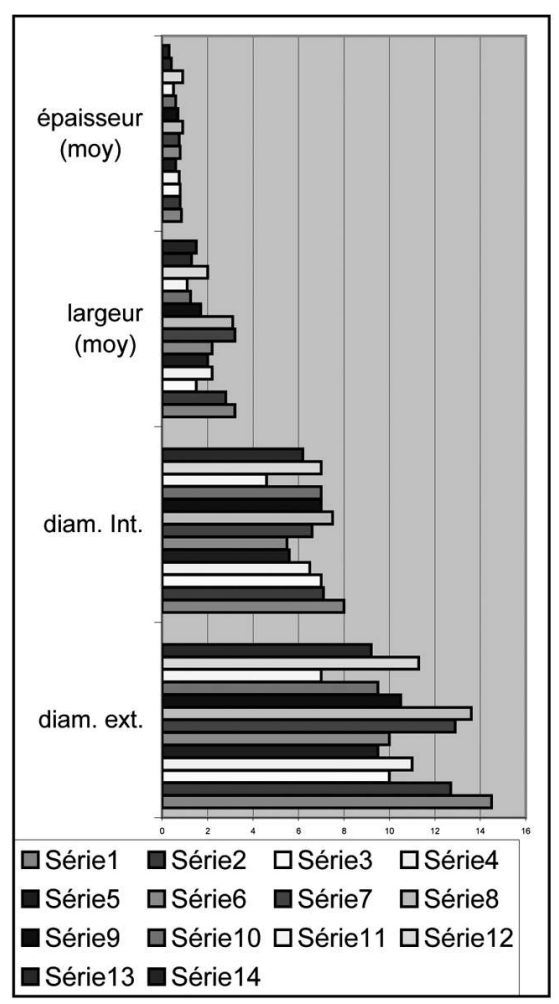

Malgré un bracelet de très petite taille $\left(\mathrm{Br} . \mathrm{n}^{\circ} 11\right)$, la moyenne des dimensions place cette série dans la catégorie des bracelets assez épais $(6,8 \mathrm{~mm})$ à très large couronne $(20,7 \mathrm{~mm})$ et à grand diamètre interne.

Trois individus sont classés dans la catégorie des anneaux disques avec un rapport diamètre externe/diamètre interne $\geq 1,8$. Ces bracelets ( $n^{\circ s} 1,7$ et 8 ) ont une largeur de couronne supérieure à $3 \mathrm{~cm}$.

Les dimensions moyennes de cette série s'apparentent très bien à celles des bracelets de la plaine de Caen (ramassage de surface). Cela est d'autant plus vrai si l'on exclut de l'ensemble le très petit bracelet $\mathrm{n}^{\circ} 11$ (Ø ext. moyen: 112,2 mm; Ø int. moyen : 75,5 mm ; Fromont, 2003).

\section{Les sections (tabl. 5)}

130 Nous avons pris en compte les critères morpho-métriques utilisés par Ivan Praud pour l'étude des bracelets des sites de Poses, d'Incarville et de Rungis (Praud, 2002):

- le type A regroupe les sections s'inscrivant dans un rectangle épais dont le rapport largeur/ épaisseur est compris entre 1 et $2 \mathrm{~cm}$;

- le type $\mathrm{B}$ comprend les sections s'inscrivant dans une forme ovalaire à rectangulaire aplatie dont le rapport largeur/épaisseur est compris entre 2,1 et $3 \mathrm{~cm}$;

- le type $\mathrm{C}$ concerne les sections s'inscrivant dans une forme ovalaire à rectangulaire mince dont le rapport largeur/épaisseur est supérieur à $3,1 \mathrm{~cm}$.

131 Les bracelets de type $C$ dominent (50\%); viennent ensuite ceux de type B (14\%). Seuls deux anneaux sont classés dans le type A. Cette grande proportion d'anneaux à large couronne de forme ovalaire à rectangulaire « mince » est constatée de façon plus large pour les bracelets bas-normands (Fromont, 2003). 
Tableau 5 : Descriptif des anneaux en schiste. Table 5: Description of the schist rings.

\begin{tabular}{|c|c|c|c|c|c|c|c|c|}
\hline anneau $\mathrm{n}^{\circ}$ & contexte & diam. ext. & diam. Int. & largeur (moy) & épaisseur (moy) & type sect. & perforation & description \\
\hline 1 & $\mathrm{~F} 7$ & 14,5 & 8 & 3,2 & 0,85 & $\mathrm{C}$ & non & schiste tacheté, claire, brillant \\
\hline 2 & $\mathrm{~F} 6$ & 12,7 & 7,1 & 2,8 & 0,8 & $\mathrm{C}$ & non & schiste ardoise, mat \\
\hline 3 & $\mathrm{~F} 25$ & 10 & 7 & 1,5 & 0,8 & $\mathrm{~A}$ & oui & shiste lité, ardoise, brillant \\
\hline 4 & $\mathrm{~F} 25$ & 11 & 6,5 & 2,2 & 0,75 & $\mathrm{~B}$ & non & schiste ardoise, mat \\
\hline 5 & $\mathrm{~F} 6$ & 9,5 & 5,6 & 2 & 0,6 & $\mathrm{C}$ & oui & shiste à Feldspath, noir \\
\hline 6 & $\mathrm{~F} 25$ (prox) & 10 & 5,5 & 2,2 & 0,8 & $\mathrm{~B}$ & non & schiste, gris claire \\
\hline 7 & $\mathrm{~F} 6$ & 12,9 & 6,6 & 3,2 & 0,75 & $\mathrm{C}$ & non & grès schisteux (briovérien) \\
\hline 8 & $\mathrm{HC}$ & 13,6 & 7,5 & 3,1 & 0,9 & $\mathrm{C}$ & non & grès schisteux (briovérien) \\
\hline 9 & $\mathrm{~F} 7$ & 10,5 & 7 & 1,7 & 0,7 & $\mathrm{~B}$ & oui & schiste tacheté, claire, brillant \\
\hline 10 & $\mathrm{~F} 6$ & 9,5 & 7 & 1,25 & 0,6 & $\mathrm{~A}$ & non & schiste tacheté, ard., brillant \\
\hline 11 & $\mathrm{~F} 6$ & 7 & 4,6 & 1,1 & 0,5 & $\mathrm{~B}$ & non & schiste tacheté, claire, brillant \\
\hline 12 & $\mathrm{~F} 6$ & 11,3 & 7 & 2 & 0,9 & $\mathrm{~B}$ & non & schiste ardoise, mat \\
\hline 13 & $\mathrm{HC}$ & 9,2 & 6,2 & 1,3 & 0,4 & $\mathrm{C}$ & non & schiste tacheté, claire, brillant \\
\hline 14 & $\mathrm{~F} 6$ & $?$ & $?$ & 1,5 & 0,3 & $\mathrm{C}$ & oui & schiste tacheté, claire, brillant \\
\hline
\end{tabular}

\section{Aspect chronologique (tabl. 6)}

Les bracelets de la frange ouest de l'aire d'influence de la culture VSG ont tendance à être plus grands, plus larges et plus épais que ceux du Bassin parisien (Fromont, 2003, t. 1, p. 55). La différence la plus nette est constatée avec la largeur de la couronne.

Dans le Bassin parisien, on pense voir une évolution chronologique dans l'accroissement du nombre de bracelets aux sections de type $C$ (aux dépens de ceux de type A) mais également dans l'apparition et la domination des anneaux en schiste provenant du Massif armoricain (schiste tacheté...). Les schistes tachetés ne seraient importés sur les sites d'habitat qu'à la fin de l'étape moyenne du VSG, voire même au cours de la dernière étape (VSG à cordon). Parallèlement, il semblerait que les couronnes des bracelets ont tendance à s'élargir vers la fin de la période. Cette évolution de la forme des bracelets et du choix de la matière première paraît démontrée sur certains sites du Bassin parisien comme celui de Rungis «site des Antes » (Praud, 2002).

Tableau 6 : Comparatif des dimensions moyennes des anneaux en schiste ; d'après I. Praud (2002) and N. Fromont (2003).

Table 6: Comparison of the average sizes of the schist rings; from I. Praud (2002) and N. Fromont (2003).

\begin{tabular}{|c|c|c|c|c|}
\hline & Belgique (82 fgts) & Nord de la France ( $88 \mathrm{fgts})$ & Rungis (42 fgts) & Poses (44 fgts) \\
\hline Diamètre int. & $63 \mathrm{~mm}$ & $69 \mathrm{~mm}$ & $62 \mathrm{~mm}$ & $62,5 \mathrm{~mm}$ \\
\hline largeur & $10 \mathrm{~mm}$ & $15 \mathrm{~mm}$ & $16 \mathrm{~mm}$ & $10,7 \mathrm{~mm}$ \\
\hline épaisseur & $6 \mathrm{~mm}$ & $7 \mathrm{~mm}$ & $4,2 \mathrm{~mm}$ & $5,5 \mathrm{~mm}$ \\
\hline & Incarville (36fgts) & B.-Normandie (?) & Plaine de Caen (?) & $\begin{array}{l}\text { FLM "Grand Champ" } \\
\text { (14fgts) }\end{array}$ \\
\hline Diamètre int. & $60 \mathrm{~mm}$ & $70,4 \mathrm{~mm}$ & $68,8 \mathrm{~mm}$ & $65,7 \mathrm{~mm}$ \\
\hline largeur & $15,5 \mathrm{~mm}$ & $20,04 \mathrm{~mm}$ & $20,7 \mathrm{~mm}$ & $20,7 \mathrm{~mm}$ \\
\hline épaisseur & $6 \mathrm{~mm}$ & $7,6 \mathrm{~mm}$ & $7,6 \mathrm{~mm}$ & $6,8 \mathrm{~mm}$ \\
\hline
\end{tabular}

134 Ce schéma ne s'applique pas forcément en Basse-Normandie, où le choix de schistes «locaux » a probablement été privilégié plutôt que sur les sites éloignés du Bassin parisien (approvisionnement dans le massif des Ardennes). La plus grande taille des bracelets bas-normands n'est-elle pas également la conséquence d'une certaine abondance de la matière première. L'exportation des schistes armoricains (notamment les schistes tachetés) à la fin de l'étape moyenne du VSG, vers le Bassin parisien a peutêtre pour conséquence une évolution de la forme des bracelets fabriqués sur ces sites d'habitat. Le modèle d'anneau à large couronne "armoricain » pourrait être alors adopté par les Néolithiques du centre du Bassin parisien. Cette hypothèse sera 
confirmée ou infirmée lorsque des sites d'habitat du Néolithique ancien de l'étape I et II du VSG, à l'attribution chronologique incontestable, seront découverts en BasseNormandie. Pour ce qui est du site de Fontenay-le-Marmion, les bracelets ne permettent pas de préciser la chronologie à l'intérieur de la culture Villeneuve-SaintGermain. Par contre, le mobilier céramique et surtout lithique placerait l'occupation plutôt dans la phase moyenne du VSG.

\section{Le bracelet en céramique (fig. 24)}

Un bracelet en céramique a été mis au jour dans la fosse sud F6. Il s'agit d'un bracelet à quatre rainures profondes $(3,2 \mathrm{~cm}$ de largeur) d'environ un centimètre d'épaisseur et de six centimètres de diamètre. Les rainures sont parallèles et espacées entre elles de 0,2 à $0,5 \mathrm{~cm}$. Sa pâte est relativement fine et comprend des inclusions de quartz.

Figure 24 : Dessin et vue du bracelet en céramique. Cliché et dessin A. Cocollos. Figure 24: Drawing and photo of the ceramic bracelet. Photo and drawing A. Cocollos.
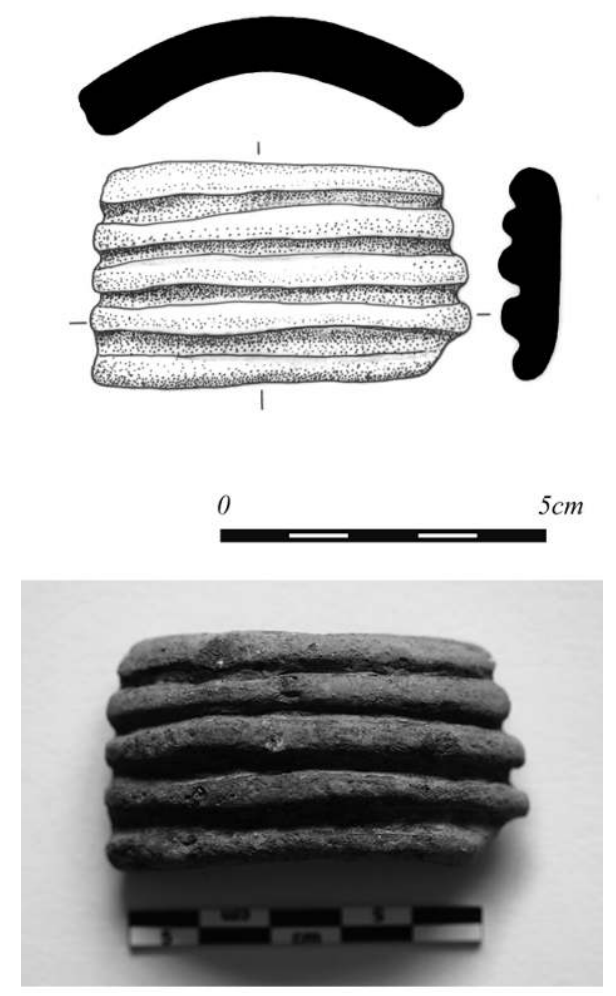

Les bracelets en céramique du Néolithique ancien sont rares dans le Nord-Ouest de la France. Pour exemple, un seul fragment de ce type de parure a été découvert sur le site de Poses "Sur la Mare ». Seuls trois bracelets rainurés dit " manchette», dont celui-ci, ont été mis au jour dans des contextes du Néolithique ancien en Normandie : un à cinq rainures à Léry (Eure) et un à quatre rainures à Poses "Clos Saint-Quentin" (Eure ; Bostyn et al., 2003). 


\section{Le macro-outillage par Caroline Hamon}

137 L'assemblage macro-lithique de Fontenay-le-Marmion "Le Grand Champ ", "Le Chemin Haussé » (Calvados) rassemble une quinzaine d'outils: vingt-cinq éclats et vingt-et-un fragments issus de seize structures (tabl. 7). Il faut leur ajouter environ cent soixante galets ou fragments naturels de plaquettes rapportés sur le site.

Tableau 7 : Catalogue des outils macro-lithiques.

Table 7: Catalogue of the macrolithic tools.

\begin{tabular}{|c|c|c|c|c|c|c|c|c|c|c|c|c|c|}
\hline st & carré & US & type & nb SA & mat prem & conserv & L & 1 & e & p & forme & section & surface active \\
\hline 5 & & 3 & herminette? & 1 & grès briovérien & fragment & 4,5 & 4,1 & 1,8 & 51 & rectang. & rect. & \\
\hline 6 & AA 9 & 1 & indét & 1 & grès briovérien & entière & 12 & 5,4 & 1,2 & 135 & trape & rect. & \\
\hline 6 & AB 4 & 2 & molette & 1 & rès violacé & fragment & 9,4 & 9,8 & 2,8 & 270 & semi-ci & rect. & concave convexe \\
\hline 6 & AA 10 & 1 & percuteur & 1 & grès briovérien & entière & 9,6 & 5,4 & 3,4 & 249 & rectang. & rect. & \\
\hline 6 & AA 1 & 1 & percuteur & 3 & grès (chaille ?) & entière & 7,8 & 7,5 & 3,8 & 256 & triang. & mi-circul. & con \\
\hline 6 & AA 1 & 2 & abraseur à main & 1 & vérien & entière & 7,4 & 4 & 2,6 & 88 & trapez. & rect. & convexe \\
\hline 6 & AA 1 & 1 & molette à main & 1 & grès violacé & entière & 7,8 & 6 & 1,8 & 100 & ovoide & semi-circul. & plano-convexe \\
\hline 7 & M1 & 3 & percuteur & 2 & grès qz & entière & 7,2 & 4,8 & 3,4 & 156 & ovoide & semi-circul. & convexe \\
\hline 11 & & & broyon & 2 & grès & re & 8 & 8 & 3,4 & 256 & circul. & rec & cor \\
\hline 15 & & & percuteur & 1 & grès grossier & entière & 8 & 8,2 & 7,1 & 734 & sphér. & circul. & convexe \\
\hline 25 & Y 13 & 5 & abraseur à main & 1 & grès orangé & fragment & 2,3 & 2,5 & 2,2 & 13 & rectang. & circul & plane \\
\hline 25 & Z 13 & 2 & indét & 1 & grès briovérien & fragment & 10,6 & 7,8 & 4,7 & 551 & rectang. & semi-circul. & plano-convexe \\
\hline 96 & & & masse & 2 & grès briovérien & entière & 17 & 9 & 11 & 2315 & ovoide & semi-circul. & \\
\hline & & & molette à main & 1 & grès violacé & fragment & 9,2 & 7,2 & 3,7 & 363 & & & pla \\
\hline & ée 55 & & molette & 1 & roche méta. & fragment & 15 & 13 & 5,8 & 1570 & ovoide & semi-circul. & plane \\
\hline
\end{tabular}

D'après X. Savary (CG 14), l'outillage du site de Fontenay-le-Marmion «Le Grand Champ » est confectionné trois variétés de grès (grès violacé, grès briovérien lité à coloration d'altération rouge et grès grossier lité à veine quartzeuse) et une dolérite verte à blanche. Épisodiquement, calcaires, silex et quartz ont été utilisés, notamment pour des opérations en percussion lancée. Quelques fragments de minerais de fer complètent la gamme des roches présentes sur le site. La majorité de ces roches employées serait d'origine locale.

139 Toutes les catégories d'outils traditionnellement rencontrées en contexte VilleneuveSaint-Germain sont représentées à un ou deux exemplaires - des meules et molettes aux polissoirs en passant par les outils de percussion et de broyage ; elles sont assez dispersées sur le site (tab. 7).

140 L'activité de broyage est représentée par deux fragments de meules ou de tables de broyage, trois fragments de molettes et trois fragments d'outils de mouture non identifiés (fig. $25 \mathrm{a}-\mathrm{c}$ ). L'un des fragments de table de broyage a été confectionné sur une plaquette de grès violacé peu épaisse. De même, une moitié de molette de facture peu soignée, obtenue également sur plaquette de grès, présente une surface active dépourvue de toute préparation par fin piquetage malgré des aspérités émoussées par l'utilisation. Ce type d'outil sur plaquette est relativement atypique en contexte Villeneuve-Saint-Germain (Hamon, 2006). La molette quasi entière en dolérite verte et blanche présente une forme ovale et une section semi-circulaire, obtenue par un piquetage fin de la surface externe. Cette molette rappelle les caractéristiques morphologiques et techniques observées sur les outils rubanés de Colombelles (Billard et al., 2004). Parmi les quelques éclats du site, quatre sont des entames de blocs bruts correspondant aux étapes initiales du façonnage (épannelage) et deux exemplaires seulement témoignent d'éventuelles étapes de ravivage de bords de meules ou de molettes. Plusieurs éclats et fragments de ravivage d'outils de mouture sont regroupés au sein de la structure 6 . Un seul broyon est ici représenté; il se rapproche des exemplaires connus dans le Néolithique ancien du Bassin parisien (Hamon, 2006). 

d'assouplissement multidirectionnel (fig. 25 c-d). Le premier objet de forme ovoïde assez régulière est en cours de reprise sur ses bords. Le second outil semble, quant à lui, soit en cours de façonnage (ébauchage) soit en cours de re-façonnage. Tous deux se distinguent par une faible durée d'utilisation. Le type de molette à main ovoïde reconnu à Fontenay-le-Marmion rappelle fortement un outil sur granite de Colombelles (Billard et al., 2004).

Figure 25 : Macro-outillage : a, d, e : molettes ; b, c : fragment de molette. Dessins $C$. Hamon. Figure 25: Macro tools: a, d, e: grinder; b, c, fragment of a grinder. Drawings C. Hamon.

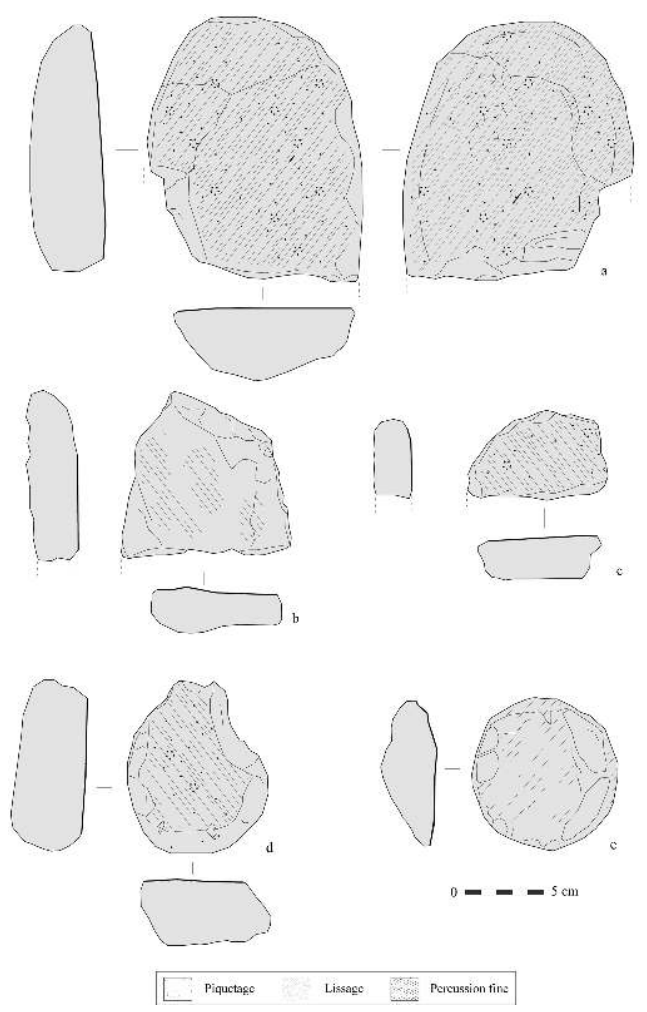

144 Trois petits fragments illustrent la présence de polissoirs à main sur le site. Le premier se présente sous la forme d'une plaquette à amorce de plage concave, le second est un fragment en cuvette et le dernier présente une surface active convexe lissée. 
(Marciny et a., 2010); comme nous le contaten à Fontenay-le-Marmion "Le Grand (Marcigny et al., 2010) ; comme nous le constatons à Fontenay-le-Marmion «Le Grand Champ ", les vestiges sont tronqués sur une épaisseur d'au moins quarante centimètres par les labours et les galeries des fouisseurs. Les structures identifiables sont surtout des fosses latérales d'habitation, principalement leur partie supérieure plus riche en rejets domestiques, celle-là même qui est arasée. Ce constat explique que plusieurs occupations de cette période, dans la campagne de Caen, n'ont été repérées que par la présence d'une unique fosse dite «isolée », probablement plus profonde que les autres et donc en partie préservée. C'est le cas à quelques kilomètres de Fontenay-le-Marmion, de la fosse Villeneuve-Saint-Germain de Tilly-la-Campagne (Giraud, en cours). Les stations de surface attribuées au Néolithique ancien, principalement de la culture VSG, ne sont pas rares sur ce territoire; elles témoignent le plus souvent de la destruction complète de structures d'habitat. Un autre handicap à l'étude de sites comparables est la faible répartition du mobilier, déjà peu abondant; les artefacts sont concentrés sur de petites surfaces à l'intérieur des fosses latérales. À Fontenay-le-Marmion « Le Grand Champ ", il suffisait de décaler la tranchée de diagnostic d'à peine un mètre vers l'ouest ou l'est pour que l'occupation néolithique passe inaperçue.

149 L'attribution chronologique précise de cet habitat pose quelques difficultés en raison de la relative pauvreté du mobilier mis au jour. Avec seulement $1,5 \mathrm{~kg}$ de céramiques exhumées nous sommes loin des six à sept kilos de tessons de poterie découverts dans certaines fosses du site de Poses «Sur la Mare» (27). Cependant, les quelques formes céramiques restituées, le bol à boutons jumelés et les grandes bouteilles, sont plutôt fréquentes dans les ensembles de la phase moyenne de la culture Villeneuve-SaintGermain. Pour le matériel lithique, la forte proportion d'outils sur lame n'est pas ici un indicateur de précocité. Par contre, la présence non négligeable d'armatures perçantes de tradition danubienne, de pièces esquillées, la faible quantité de grattoirs et surtout, l'absence de certains outils comme les tranchets placeraient l'ensemble également dans la phase moyenne du VSG. Certains éléments de parure, notamment les quelques anneaux minces à couronnes plutôt larges semblent exclure une attribution de l'occupation à la phase ancienne du Villeneuve-Saint-Germain. Une datation par le radiocarbone, réalisée sur des résidus calcinés retrouvés dans un vase de la fosse sud,

Revue archéologique de l'Ouest, 29 | 2012 
confirmerait un rattachement à la phase moyenne avec un âge calibré de 5044 à 4808 avant notre ère (Lyon-2596, date 14C BP : 6045 \pm 40 BP).

L'hypothèse d'une habitation isolée, comme elle a été proposée pour la maison VSG à cordon de Mondeville, n'est pas vérifiable ici en raison de l'exiguïté de l'emprise du chantier et de la présence au sud d'une importante voie antique. L'abondance des pièces lithiques en surface de la parcelle de terrain, hors emprise, abonde plutôt pour une implantation plus vaste.

Un grand merci à Nicolas Coulthard pour la traduction du résumé et des légendes de cet article.

\section{BIBLIOGRAPHIE}

AUGEREAU A., 2004 - L'industrie du silex $d u v^{e}$ au Ive millénaire dans le sud-est du Bassin parisien : Rubané, Villeneuve-Saint-Germain, Cerny et groupe de Noyen, Paris, MSH, 2004.

AUXIETTE G., 1989 - « Les bracelets néolithiques dans le Nord de la France, la Belgique et l'Allemagne rhénane », Revue archéologique de Picardie, $\mathrm{n}^{\text {os }} 1 / 2$, p. 13-44.

BILLARD C., 2001-2003 - Colombelles « Le Lazzaro » (Calvados). Un habitat du Néolithique ancien dans la plaine de Caen. Rapports de fouille DRAC Basse Normandie, conseil général du Calvados, Ville de Colombelles.

BILLARD C., ALIX P., BONNABEL L., BONNARDIN S., BOSTYN F., CASPAR J.-P., DEGOBERTIÈRE S., DIETSCH-SELLAMI M.-F., HAMON C., MARCOUX N. et MARGUERIE D., 2004 - « Le site d'habitat du Néolithique ancien de Colombelles « le Lazarro » (Calvados) - Présentation préliminaire », Internéo, n 5, p. 29-33.

BLANCHARD N. et FORRÉ P., 2003 - « Géologie et géographie de l'opalite en Centre-Ouest », in Feuillets mensuels de la Société nantaise de préhistoire, $\mathrm{n}^{\circ} 411,47^{\mathrm{e}}$ année, juin 2003, p. 34-39.

BLANCHET S., FORRÉ P., FROMONT N., HAMON C., HAMON G., 2010 - « Un habitat du Néolithique ancien à Betton « Pluvignon » (Ille et Vilaine) », in Billard C. et LeGris M., Premiers Néolithiques de l'Ouest : cultures, réseaux, échanges des premières sociétés néolithiques à leur expansion, actes du colloque interrégional sur le Néolithique, Le Havre, 2007, PUR, p. 15-39.

BOSTYN F., HACHEM L., LANCHON Y., 1991 - « Le site néolithique de Jablines « La Pente de Croupeton » (Seine et Marne) : premiers résultats ", Actes du colloque néolithique de Chalon-sur-marne, ARPEPP, p. 45-81.

BOSTYN F., 1994 - Caractérisation des productions et de la diffusion des industries lithiques du groupe néolithique du Villeneuve-Saint-Germain. Doctorat de l'université de Paris X-Nanterre. BOSTYN F., PRAUD I., 2000 - « Le site néolithique de Raillencourt-Sainte-Olle « Le Grand Camp » (Nord) », Internéo, nº 3, p. 119-130.

BOSTYN F. (dir.), 2003 - « Néolithique ancien de Haute-Normandie : le village Villeneuve-SaintGermain de Poses « Sur la Mare » et les sites de la Boucle du Vaudreuil. Travaux 4 », Bulletin de la Société préhistorique française. 
BURNEZ-LANOTTE L. et CASPAR J.-P., avec la coll. de VANGUESTAINE M., 2005 - « Technologie des anneaux en schiste dans le groupe de Blicquy/Villeneuve-Saint-Germain à Vaux-et-Borset (Hesbaye, Belgique) : interférences et sous-systèmes techniques ", Bulletin de la Société préhistorique française, t. $102, n^{\circ} 3$, p. 551-596.

CASSEN S., HAUDREN C., HINGUANT S., LANNUZEL G. et MARCHAND G., 1998 - « L'habitat Villeneuve-SaintGermain du Haut-Mée (Saint-Étienne-en-Coglès, Ille-et-Vilaine) », Bulletin de la Société préhistorique française, t.95, p.41-75.

CASPAR J.-P., BURNEZ-LANOTTE L., 2003 - « Gestion des matériaux siliceux dans les premières communautés danubiennes (culture à céramique linéaire et groupe de Blicquy-Villeneuve-SaintGermain) à Vaux-et-Borset (Hesbaye, Belgique) », in BURNEZ-LANOTTE L. (dir.), Production and Management of Lithic Materials in the European Linearbandkeramik/Gestion des matériaux lithiques dans le Rubané européen, Actes du XIVe congrès UISPP, université de Liège, Belgique, 2-8 septembre 2001, B.A.R. International Series 1200, Oxford, p. 51-58.

CASPAR J.P, FÉRAY P. et MARTIAL E., 2003 - « Identification et reconstitution des traces de teillage des fibres végétales au Néolithique », Bulletin de la Société préhistorique française, 2005, t. 102, nº 4.

CHANCEREL A., DESLOGES J., DRON J.-L. et SAN JUAN G., 1992 - « Le début du Néolithique en BasseNormandie », Revue archéologique de l'Ouest, suppl. n 5, p. 153-173.

CHANCEREL A., GHESQUIÈRE E., LEPAUMIER H., FORFAIT N. et LECLERC G., 1995 - « Nouvelles implantations du groupe de Villeneuve-Saint-Germain en Basse-Normandie ", Revue archéologique de l'Ouest, suppl. $\mathrm{n}^{\circ}$ 7, p. 43-54.

CHANCEREL A., MARCIGNY C. et GHESQUIERE E., 2006 - Le plateau de Mondeville (Calvados) du Néolithique à l'âge du Bronze, Paris, MSH, 2006.

COUTARD S., 1998 - Panorama des silex jurassiques bas-normands. Essai de caractérisation et répartition, mémoire de maîtrise de l'université de Caen, département de géologie, 83 p.

DEROUIN A. (dir.), 1999 - Notice explicative, carte géologique de la France 1/50000, feuille de Mézidon (146), BRGM, Orléans.

FECHNER K. et LANGHOR R., 1998 - « Utilisation des sédiments, fonction des fosses et choix des sols dans l'habitat rubané. État de la question », in CAUWE N. et VAN BERG P.-L. (éd.), XXIIIe colloque interrégional sur le Néolithique : anthropologie et préhistoire, Bruxelles, p. 121-140.

FEDOROFF N. et COURTY M.-A., 1994 - « Organisation du sol aux échelles microscopiques », in BONNEAU M. et Souchier B. (éd.), Pédologie, 2. Constituants et propriétés des sols, $2^{\mathrm{e}}$ édition, Paris, p. 349-375. FROMONT N., 2003 - Les anneaux en matériaux lithiques de Basse-Normandie, Université de Caen ; MAE (UMR 7041), septembre 2003, t. 1 et 2.

GIAZZON D., MARCIGNY C. et GHESQUIERE E., 2002 - « Un nouveau site de production de bracelets en schiste dans le nord de la Sarthe : Arçonnay "Le Parc Saint-Gilles" », Bulletin de la société préhistorique française, $n^{\circ} 99$ (4), p. 825-828.

GIRAUD P. et JUHEL L., 2004 - « L'habitat Villeneuve-Saint-Germain "Le Grand Champ" (Calvados) », Internéo, $\mathrm{n}^{\circ}$ 5, p. 35-43.

MARCIGNY C., GHESQUIÈRE E., GIAZZON D. et GAUMÉ E., 1999 - « Un site de production de parures en schiste dans le nord du département de la Sarthe à Champfleur "Bois de Barrée" ", Bulletin de la société préhistorique française, $\mathrm{n}^{\circ} 96$ (4), p. 639-642. 
MARCIGNY C., GHESQUIÈRE E., JUHEL L. et CHARRAUD F., 2010 - « Entre Néolithique ancien et Néolithique moyen en Normandie et dans les îles Anglo-Normandes, parcours chronologique ", in BILLARD C. et LEGRIS M., Premiers Néolithiques de l'Ouest : cultures, réseaux, échanges des premières sociétés néolithiques à leur expansion, actes du colloque interrégional sur le Néolithique, Le Havre, 2007, PUR, p. 117-162.

HAMON C., 2006 - Broyage et abrasion au Néolithique ancien. Caractérisation technique et fonctionnelle des outillages en grès du Bassin parisien, BAR International Reports S1551, Oxford, 342 p.

JAN D., 2010 - Les céramiques néolithiques à dégraissant végétal en Basse-Normandie : Études des pâtes, identification des végétaux et protocole expérimental, Vol.1 : Élaboration du corpus, étude pétrographique des pâtes et observation des inclusions végétales, Mémoire de master $1:$ « Archéologie et environnement ", université Paris 1, 95 p.

JAN D., 2011 - Les céramiques néolithiques à dégraissant végétal en Basse-Normandie : Etudes des pâtes, identification des végétaux et protocole expérimental, Vol.2 : Protocole expérimental et identification du dégraissant végétal, Mémoire de master 2 : «Archéologie et environnement », université Paris 1, 72 p.

JUHEL L., GHESQUIERE E. et MARCIGNY C., 2006 - « L'abri sous roche néolithique moyen de la Jupinerie (Omonville-la-Petite, Manche) », Internéo 6, Paris.

PRAUD I., 2002 - « Les éléments de parure », in BOSTYN, F. (dir.), Néolithique et protohistoire du site des Antes de Rungis (Val-de-Marne), Ed. Artcom', p. 91-102.

SLIVA R.-J. et KEELEY L.-H., 1994 - « "Frits” and Specialized Hide Preparation in the Belgian Early Neolitic", Journal of Archeological Science, 21, p. 91-99.

SIMONIN D., 1996 - « Les habitats néolithiques d'Echilleuses (Loiret). Analyse spatiale des documents archéologiques », Revue archéologique du Loiret, Édition de la fédération archéologique du Loiret, $n^{\circ} 21-22$.

\section{RÉSUMÉS}

L'unité d'habitation du Néolithique ancien (VSG) de Fontenay-le-Marmion « Le Grand Champ » a été mise au jour lors d'une opération d'archéologie préventive du conseil général du Calvados. Ce site, en contexte de plateau, est localisé à une dizaine de kilomètres au sud de l'agglomération caennaise. Les structures de cet habitat sont difficilement identifiables sans une étude précise de la répartition spatiale du mobilier. Elles correspondent à trois fosses latérales d'habitation, deux fosses sud et une fosse nord ainsi qu'à quelques petites excavations interprétables comme les trous de poteaux d'une maison. Le mobilier provient presque exclusivement des fosses latérales d'habitation ; sans être abondant, il constitue un ensemble cohérent. Le matériel lithique en silex est le plus conséquent avec une production d'outils en silex principalement sur lame. Ce site a livré très peu de formes céramiques; les pièces identifiées s'insèrent parfaitement dans les ensembles classiques de la culture VSG (grandes bouteilles, vases à profil en U ou à profil dit « en bombe »). Le macro-outillage en roche dure est également bien représenté. La fabrication de bracelet en schiste sur place est attestée par la présence de plusieurs pièces de la chaîne opératoire. L'étude tracéologique démontre également que deux grattoirs ont été utilisés pour travailler de la roche tendre, peut-être du schiste. L'étude des différents types de mobilier et une datation par le radiocarbone convergent pour placer cette occupation dans la phase moyenne du VSG ; entre le village rubané récent et VSG ancien de Colombelles (14) et la maison VSG à cordon de Mondeville (14). 
The early Neolithic habitation (VSG) at Fontenay-le-Marmion «Le Grand Champ », was brought to light during an excavation both funded and carried out by the conseil général du Calvados (Calvados departmental Council) prior to development. The site is situated on a plateau, approximately $10 \mathrm{~km}$ south of Caen. The features revealed are difficult to identify without precise spatial analysis of artefacts. They comprise three pits lateral to the habitation, two to the north and one to the south, with a few small cuts than can be interpreted as post holes. The artefacts, found almost exclusively in the lateral pits, make up a coherent - if not abundant assemblage. Flint lithics are the most numerous with a production of mainly blade based tools. The site has yielded few pottery forms; the elements identified fit in perfectly with the classical assemblages of the VSG culture (large bottles, round based recipients or with spherical profiles). Hard stone macrotools are also well represented. The manufacture of schist bracelets in situ is attested by the presence of different elements of the production chain. Microwear analysis also shows that two scrapers have been used to work a soft stone, perhaps schist.

\section{INDEX}

Mots-clés : Calvados, plaine de Caen, habitat, industrie lithique, poteries, parure en schiste, bracelet en céramique, Néolithique, micromorphologie, macro-outillage, fosse

Keywords : Calvados, Caen plain, Early Neolithic, habitat, adjacent ditches, lithic industry, pottery, schist bangle, ceramic bracelet, macro tools, micromorphology, petrography, use wear analysis

\section{AUTEURS}

\section{PIERRE GIRAUD}

Attaché de conservation du patrimoine - Service archéologie, DGA Jeunesse, Culture et Territoires - Conseil général du Calvados - pierre.giraud@calvados.fr.

\section{LAURENT JUHEL}

Inrap Bretagne et UMR 6566 CReAAH - laurent.juhel@inrap.fr.

\section{CAROLINE HAMON}

Chargée de recherche CNRS - UMR 7041 ArscAn Protohistoire européenne - Maison de l'archéologie et de l'ethnologie - 21 allée de l'Université F-92023 Nanterre cedex caroline.hamon@mae.cnrs.fr.

\section{CÉCILE GERMAIN}

Attachée de conservation du patrimoine - Service archéologie, DGA Jeunesse, Culture et Territoires - Conseil général du Calvados - cecile.germain@calvados.fr.

\section{XAVIER SAVARY}

Attaché de conservation du patrimoine - Service archéologie, DGA Jeunesse, Culture et Territoires - Conseil général du Calvados - xavier.savary@calvados.fr.

\section{DENIS JAN}

Contractuel Conseil général du Calvados et étudiant en master 2 à l'université de Paris 1. 